\title{
Reconstruction of Multidimensional Signals From Irregular Noisy Samples
}

\author{
Alessandro Nordio, Member, IEEE, Carla-Fabiana Chiasserini, Member, IEEE, and \\ Emanuele Viterbo, Senior Member, IEEE
}

\begin{abstract}
We focus on a multidimensional field with uncorrelated spectrum and study the quality of the reconstructed signal when the field samples are irregularly spaced and affected by independent and identically distributed noise. More specifically, we apply linear reconstruction techniques and take the mean-square error (MSE) of the field estimate as a metric to evaluate the signal reconstruction quality. We find that the MSE analysis could be carried out by using the closed-form expression of the eigenvalue distribution of the matrix representing the sampling system. Unfortunately, such distribution is still unknown. Thus, we first derive a closed-form expression of the distribution moments, and we find that the eigenvalue distribution tends to the Marčenko-Pastur distribution as the field dimension goes to infinity. Finally, by using our approach, we derive a tight approximation to the MSE of the reconstructed field.
\end{abstract}

Index Terms-Field reconstruction, irregular sampling, random matrix theory, wireless sensor networks.

\section{INTRODUCTION}

W E address the important issue of reconstructing a multidimensional signal from a collection of samples that are noisy and not uniformly spaced. As a case study, we consider a wireless sensor network for environmental monitoring, where the nodes sensing the physical phenomenon (hereinafter also called field) are randomly deployed over the area under observation. The sensors sample a $d$-dimensional spatially finite physical field, where $d$ may take into account spatial dimensions as well as the temporal dimension. Examples of such fields are pressure or temperature, on a four-dimension domain, i.e., three spatial coordinates plus the time dimension. A spatially finite physical field is not band-limited; however, it admits an infinite Fourier series expansion. Here, we consider a finite approximation of the physical field obtained by truncating such series, assuming that the contribution of the truncated terms is negligible.

In our case study, we assume that the measured samples are transferred from the sensors to a common data-collecting unit, the so-called sink node, which is in charge of reconstructing the field. Distributed systems, where in-network processing, is performed are out of the scope of this work. We do not deal

Manuscript received August 22, 2007; revised April 9, 2008. First published May 23, 2008; last published August 13, 2008 (projected). The associate editor coordinating the review of this manuscript and approving it for publication was Prof. Qing Zhao. This work was supported by MIUR through the MEADOW project.

A. Nordio and C.-F. Chiasserini are with the Department of Electronic Engineering, Politecnico di Torino, 10129 Turin, Italy (e-mail: alessandro.nordio@polito.it; carla.chiasserini@polito.it).

E. Viterbo is with the DEIS, Università della Calabria, 87036 Rende (CS), Italy (e-mail: viterbo@deis.unical.it).

Digital Object Identifier 10.1109/TSP.2008.925953 with issues related to information transport and, thus, we assume that all samples are correctly received at the sink node. The field samples, however, are corrupted by additive independent and identically distributed (i.i.d.) noise, due to quantization, round-off errors, or quality of the sensing device. Furthermore, the sampling points are known at the sink node, because i) either sensors are located at predefined positions or their position can be estimated through a localization technique [1], and ii) the sampling time is either periodic or included in the information sent to the sink.

Several efficient and fast algorithms have been proposed to numerically reconstruct or approximate a signal in such setting, which amount to the solution of a linear system (see [2] and [3] and references therein). A widely used technique consists in processing the sensors' measures by means of a linear filter, which is a function of the system parameters known at the sink. We observe that the following two major factors affect the linear reconstruction:

i) the given machine precision, which may prevent the reconstruction algorithm from performing correctly and may lead to a non-negligible probability of reconstruction failure [3];

ii) the noise level affecting the sensors' measurements. In the latter case, a measure of reconstruction accuracy is given by the mean-square error (MSE) of the field estimate. In [4] and [5], we have found that these issues could be studied by using the eigenvalue distribution of the reconstruction matrix; however, obtaining such a distribution is still an open problem. In this work, we first extend the system model and the problem formulation presented in [5] to the case of multidimensional fields (Section III). Then, we derive a closed-form expression of the moments of the eigenvalue distribution, through asymptotic analysis (Section V). By using the moments expressions, we prove that the eigenvalue distribution of the matrix representing the sampling system tends to the Marčenko-Pastur distribution [6] as the field dimension $d \rightarrow \infty$ (Section VI).

We apply our results to the study of the MSE of the field estimate, when the sensors measurements are noisy and the reconstruction at the sink is performed through linear filtering.

We generalize the MSE expressions to the multidimensional case (with finite $d$ ), and we show that, by using the Marčenko-Pastur distribution instead of the actual eigenvalue distribution, we obtain an approximation to the MSE of the reconstructed field which is very tight for $d \geq 3$ (Section VII).

Before providing a detailed description of our analysis, in the next section we discuss some related studies and highlight our main contributions with respect to previous work. 


\section{RELATED WORK AND MAIN CONTRIBUTIONS}

Relevant to our work is the literature on spectral analysis, where, however, several studies deal with regularly sampled signals (e.g., [7] and references therein). An excellent guide to irregular sampling is [8], which presents a large number of techniques, algorithms, and applications. Reconstruction techniques for irregularly or randomly sampled signals can be found in [3], [9], and [10], just to name few. In particular, Feichtinger and Gröchenig in [10] provide an error analysis of an iterative reconstruction algorithm taking into account round-off errors, jitters, truncation errors and aliasing. From the theoretical point of view, irregular sampling has been studied in [3], [9]-[14] and references therein.

In the context of sensor networks, efficient techniques for spatial sampling are proposed in [15] and [16]. In particular, in [16], an adaptive sampling is described, which allows the central data collector to vary the number of active sensors, i.e., samples, according to the desired resolution level. Data acquisition is also studied in [17], where the authors consider a unidimensional field, uniformly sampled at the Nyquist frequency by low precision sensors. The authors show that the number of samples can be traded off with the precision of sensors. The problem of the reconstruction of a bandlimited signal from an irregular set of samples at unknown locations is addressed in [18]. There, different solution methods are proposed, and the conditions for which there exist multiple solutions or a unique solution are discussed. Differently from [18], we assume that the sink can either acquire or estimate the sensor locations and that sensors are randomly deployed.

The field reconstruction at the sink node with spatial and temporal correlation among sensor measures is studied, for instance, in [19]-[23]. Other interesting studies can be found in [24] and [25], which address the perturbations of regular sampling in shift-invariant spaces [24] and the reconstruction of irregularly sampled images in presence of measurement noise [25].

We point out that our main contribution with respect to previous work on signal sampling and reconstruction is the probabilistic approach we adopt to analyze the quality level of a signal reconstructed from a set of irregular, noisy samples. Our analysis, however, applies to sampling systems where the field reconstruction is performed in a centralized manner. Finally, we highlight that our previous work [5] assumes that sensors are uniformly distributed over the spatial observation interval and may be displaced around a known average location. The effects of noisy measures and jittered positions are analyzed when linear reconstruction techniques are employed. However, only the unidimensional case is studied, and semianalytical derivations of the MSE of the reconstructed field are obtained. In [26], instead, sensors are assumed to be fixed, and the objective is to evaluate the performance of a linear reconstruction technique in the presence of quasi-equally spaced sensor layouts.

\section{A. Main Results}

The goal of this work is to provide an analytical study on the reconstruction quality of a multidimensional physical field, with uncorrelated spectrum. The field samples are i) irregularly spaced, since they are gathered by a randomly deployed sensor network and ii) affected by i.i.d. noise. The sink node receives the field samples and runs the reconstruction algorithm in a centralized manner. Our major contributions with respect to previous work are as follows.

1) Given a $d$-dimensional problem formulation, we obtain analytical expressions for the moments of the eigenvalue distribution of the reconstruction matrix. Using the expressions of the moments, we show that the eigenvalue distribution tends to the Marčenko-Pastur distribution [6] as the field dimension $d \rightarrow \infty$.

2) We apply our results to the study of the quality of a reconstructed field and derive a tight approximation to the MSE of the estimated field.

\section{PRELIMINARIES}

We first present the multidimensional formulation of our reconstruction problem. Then, we give some background on linear reconstruction techniques and generalize to the multidimensional case some results previously obtained in the unidimensional case [5]. Finally, we highlight the main steps followed in our study.

Notation: Lower case bold letters denote column vectors, while upper case bold letters denote matrices. We denote the $(h, k)$ th entry of the matrix $\mathbf{X}$ by $(\mathbf{X})_{h, k}$, the transpose of $\mathbf{x}$ by $\mathbf{x}^{\mathrm{T}}$, and the conjugate transpose of $\mathbf{x}$ by $\mathbf{x}^{\dagger}$. The identity matrix is denoted by $\mathbf{I}$. Finally, $\mathbb{E}[x]$ is the average of $x$ and subscripts to the average operator specify the variable with respect to which the average is taken.

\section{A. Irregular Sampling of Multidimensional Signals}

Let us consider a $d$-dimensional, spatially-finite physical field $(d \geq 1)$, where $r$ sensors are located in the hypercube $\mathcal{H}=$ $\left\{\mathbf{x} \mid \mathbf{x} \in[0,1)^{d}\right\}$ and measure the value of the field. We assume that the sensor sampling points are known. At first, we consider that they are deterministic, then we will assume that they are i.i.d. random variables uniformly distributed in the hypercube $\mathcal{H}$.

When observed over a finite region, a $d$-dimensional physical field $s(\mathbf{x})$ with finite energy $E_{s}$ admits an infinite $d$-dimensional Fourier series expansion with coefficients $\tilde{a}_{\ell}$, such that $E_{s}=\sum_{\ell_{1}, \ldots, \ell_{d}=-\infty}^{+\infty}\left|\tilde{a}_{\ell}\right|^{2}$ where $\boldsymbol{\ell}=\left[\ell_{1}, \ldots, \ell_{d}\right]$ is a vector of integers and $\ell_{m}, m=1, \ldots, d$ represents the index of the expansion along the $m$ th dimension. We truncate the expansion to $2 M+1$ terms per dimension where $M$ is such that $\sum_{\ell_{1}, \ldots, \ell_{d}=-\infty}^{-M-1}\left|\tilde{a}_{\ell}\right|^{2}+\sum_{\ell_{1}, \ldots, \ell_{d}=M+1}^{+\infty}\left|\tilde{a}_{\ell}\right|^{2} \ll E_{s}$ Therefore, one can think of $M$ as the approximate one-sided bandwidth (per dimension) of the field, which can be approximated over the finite region $\mathcal{H}$ as

$$
s(\mathbf{x})=(2 M+1)^{-d / 2} \sum_{\boldsymbol{\ell}} a_{\nu(\boldsymbol{\ell})} \mathrm{e}^{\mathrm{j} 2 \pi \mathbf{x}^{\mathrm{T}} \boldsymbol{\ell}}
$$

where the term $(2 M+1)^{-d / 2}$ is a normalization factor and $\sum_{\boldsymbol{\ell}}$ represents a $d$-dimensional sum over the vector $\ell$, with $\ell_{m}=$ $-M, \ldots, M$. Also, $a_{\nu(\ell)}=\tilde{a}_{\ell}$ and the function

$$
\nu(\ell)=\sum_{m=1}^{d}(2 M+1)^{m-1} \ell_{m}
$$


$-\left(\left((2 M+1)^{d}-1\right) / 2\right) \leq \nu(\ell) \leq+\left(\left((2 M+1)^{d}-1\right) / 2\right)$ maps the vector $\boldsymbol{\ell}$ onto a scalar index. Note that, while $\tilde{a}_{\boldsymbol{\ell}}$ has a vectorial index, $a_{\nu(\ell)}$ has a scalar index and it has been introduced to simplify the notation. As an example, for $d=2$ and $M=1$, we have $\nu(\ell)=3 \ell_{1}+\ell_{2}$ and $s\left(x_{1}, x_{2}\right)=$ $(1 / 3) \sum_{\ell_{1}=-1}^{1} \sum_{\ell_{2}=-1}^{1} a_{3 \ell_{2}+\ell_{1}} \mathrm{e}^{\mathrm{j} 2 \pi\left(x_{1} \ell_{1}+x_{2} \ell_{2}\right)}$.

Let $\mathcal{X}=\left\{\mathbf{x}_{1}, \ldots, \mathbf{x}_{r}\right\}$, with $\mathbf{x}_{q}=\left[x_{q, 1}, \ldots, x_{q, d}\right]^{\mathrm{T}} \in \mathcal{H}$, $q=1, \ldots, r$, be the set of sampling points, and $\mathbf{s}=$ $\left[s_{1}, \ldots, s_{r}\right]^{\mathrm{T}}, s_{q}=s\left(\mathbf{x}_{q}\right)$, the values of the corresponding field samples. Following [3], we write the vector of field values $\mathbf{s}$ as a function of the spectrum:

$$
\mathbf{s}=\mathbf{G}_{d}^{\dagger} \mathbf{a}
$$

where $\mathbf{a}$ is a vector of size $(2 M+1)^{d}$, whose $\nu(\boldsymbol{\ell})$ th entry is given by $a_{\nu(\ell)}$, and $\mathbf{G}_{d}$ is the $(2 M+1)^{d} \times r$ matrix

$$
\left(\mathbf{G}_{d}\right)_{\nu(\boldsymbol{\ell}), q}=(2 M+1)^{-d / 2} \mathrm{e}^{-\mathrm{j} 2 \pi \mathbf{x}_{q}^{\mathrm{T}} \boldsymbol{\ell}} .
$$

In general, the entries of a can be correlated with covariance matrix $\mathbb{E}\left[\mathbf{a a}^{\dagger}\right]=\sigma_{a}^{2} \mathbf{C}_{a}$, and $\operatorname{Tr}\left\{\mathbf{C}_{a}\right\}=(2 M+1)^{d}$. In the following, we restrict our analysis to the class of fields characterized by $\mathbb{E}\left[\mathbf{a a}^{\dagger}\right]=\sigma_{a}^{2} \mathbf{I}$. If the sensor measurements, $\mathbf{p}=$ $\left[p_{1}, \ldots, p_{r}\right]^{\mathrm{T}}$, are noisy, then the relation between sensors' samples and field spectrum can be written as

$$
\mathbf{p}=\mathbf{s}+\mathbf{n}=\mathbf{G}_{d}^{\dagger} \mathbf{a}+\mathbf{n}
$$

where the noise is represented by the $r$-size, zero-mean random vector $\mathbf{n}$, with covariance matrix $\mathbb{E}\left[\mathbf{n n}^{\dagger}\right]=\sigma_{n}^{2} \mathbf{I}_{r}$. We define the signal-to-noise ratio on the measure as $\mathrm{SNR}_{m} \triangleq \sigma_{a}^{2} / \sigma_{n}^{2} \triangleq$ $1 / \alpha$.

\section{B. Sampling Rate}

Following [5], we introduce the parameter $\beta$ defined as

$$
\beta=\frac{(2 M+1)^{d}}{r} .
$$

This parameter represents the ratio between the number of harmonics used for the field reconstruction and the number of sensors sampling the field. In the following, we consider $\beta \in[0,1)$. Notice that for fixed $\beta$ and $M$, the number $r$ of samples exponentially increases with $d$.

\section{Previous Results on Reconstruction Quality}

Given an estimate â of the field spectrum a, the reconstructed signal is

$$
\hat{s}(\mathbf{x})=(2 M+1)^{-d / 2} \sum_{\boldsymbol{\ell}} \hat{a}_{\nu(\boldsymbol{\ell})} \mathrm{e}^{\mathrm{j} 2 \pi \mathbf{x}^{\mathrm{T}} \boldsymbol{\ell}} .
$$

As reconstruction performance metric, we consider the MSE of the field estimate, which, for any given set of sampling points $\mathcal{X}$, is defined as

$$
\operatorname{MSE}_{\mathcal{X}}=\underset{\mathbf{a}, \mathbf{n}}{\mathbb{E}} \int_{\mathcal{H}}|\hat{s}(\mathbf{x})-s(\mathbf{x})|^{2} \mathrm{~d} \mathbf{x}=\frac{\mathbb{\mathbf { a } , \mathbf { n }}\|\mathbf{\mathbf { a }}-\mathbf{a}\|^{2}}{(2 M+1)^{d}}
$$

where the average is taken with respect to the subscripted random vectors. Note that (7) still assumes that the sampling points are deterministic; this assumption will be removed later in the paper.

For linear models such as (4), several estimation techniques based on linear filtering are available in the literature [27]. We employ a filter $\mathbf{B}$ such that the estimate of the field spectrum is given by the linear operation

$$
\hat{\mathbf{a}}=\mathbf{B}^{\dagger} \mathbf{p}
$$

where $\mathbf{B}$ is an $r \times(2 M+1)^{d}$ matrix. In particular, we consider the linear filter providing the best performance in terms of MSE, i.e., the linear minimum MSE (LMMSE) filter ${ }^{1}$ [27]

$$
\mathbf{B}=\mathbf{G}_{d}^{\dagger}\left(\mathbf{R}_{d}+\alpha \mathbf{I}\right)^{-1}
$$

where $\mathbf{R}_{d}=\mathbf{G}_{d} \mathbf{G}_{d}^{\dagger}$.

From now on, we carry out our analysis under the assumption that the elements of the set $\mathcal{X}$ are independent random vectors, with i.i.d. entries, uniformly distributed in the hypercube $\mathcal{H}$.

In [5], we have shown that a simple and effective tool to evaluate the performance of large finite systems is asymptotic analysis. We computed the MSE by letting the field number of harmonics and the number of samples grow to infinity, while their ratio $\beta=(2 M+1)^{d} / r$ is kept constant. We observed the validity of asymptotic analysis results, even for small values of $M$ and $r$. Similarly, here we consider as performance metric the asymptotic average MSE, normalized to $\sigma_{a}^{2}$ :

$$
\mathrm{MSE}_{\infty}=\lim _{M, r \rightarrow+\infty} \frac{1}{\sigma_{a}^{2}} \underset{\mathcal{X}}{\mathbb{E}}\left[\mathrm{MSE}_{\mathcal{X}}\right]
$$

where $\beta$ below the limit denotes the ratio which is kept constant. In (10), the average is over all possible realizations of the set $\mathcal{X}$. Using (7)-(9), and the above definitions, in Appendix E we show that, in the case of the LMMSE

$$
\mathrm{MSE}_{\infty}=\underset{\lambda_{d, \beta}}{\mathbb{E}}\left[\frac{\alpha \beta}{\lambda_{d, \beta}+\alpha \beta}\right]
$$

where $\lambda_{d, \beta}$ is a random variable with probability density function (pdf) $f_{d, \beta}(x)$, distributed as the asymptotic eigenvalues of $\mathbf{T}_{d}=\beta \mathbf{R}_{d}=\beta \mathbf{G}_{d} \mathbf{G}_{d}^{\dagger}$. The subscripts $d$ and $\beta$ of $\lambda$ indicate that the distribution of the asymptotic eigenvalues of $\mathbf{T}_{d}$ depends on both the field dimension $d$ and the parameter $\beta$.

The matrix $\mathbf{T}_{d}$ plays an important role in our analysis; in the following, we introduce some of its properties. In the unidimensional case $(d=1), \mathbf{T}_{1}$ is a $(2 M+1) \times(2 M+1)$ Hermitian Toeplitz matrix given by

$$
\mathbf{T}_{1}=\mathbf{T}_{1}^{\dagger}=\left(\begin{array}{cccc}
t_{0} & t_{1} & \cdots & t_{2 M} \\
t_{-1} & t_{0} & \cdots & t_{2 M-1} \\
& & \ddots & \\
t_{-2 M} & t_{-2 M+1} & \cdots & t_{0}
\end{array}\right)
$$

${ }^{1}$ Notice that when the covariance matrix of $\mathbf{a}$ is known, the LMMSE filter generalizes to $\left(\mathbf{G}_{d}^{\dagger} \mathbf{C}_{a} \mathbf{G}_{d}+\alpha \mathbf{I}\right)^{-1} \mathbf{G}_{d}^{\dagger} \mathbf{C}_{a}$. 
where $\left(\mathbf{T}_{1}\right)_{\ell, \ell^{\prime}}=t_{\ell-\ell^{\prime}}=(1 / r) \sum_{q=1}^{r} \exp \left(-\mathrm{j} 2 \pi\left(\ell-\ell^{\prime}\right) x_{q}\right)$, $\ell, \ell^{\prime}=-M, \ldots, M$. For $d \geq 2, \mathbf{T}_{d}$ can be defined recursively as a $(2 M+1)^{d} \times(2 M+1)^{d}$ Hermitian block Toeplitz matrix with non-Hermitian Toeplitz blocks

$$
\mathbf{T}_{d}=\left(\begin{array}{cccc}
\mathbf{B}_{0} & \mathbf{B}_{1} & \cdots & \mathbf{B}_{2 M} \\
\mathbf{B}_{-1} & \mathbf{B}_{0} & \cdots & \mathbf{B}_{2 M-1} \\
\vdots & \vdots & & \vdots \\
\mathbf{B}_{-2 M} & \mathbf{B}_{-2 M+1} & \cdots & \mathbf{B}_{0}
\end{array}\right)
$$

where

$$
\left(\mathbf{T}_{d}\right)_{\nu(\boldsymbol{\ell}), \nu\left(\boldsymbol{\ell}^{\prime}\right)}=\frac{1}{r} \sum_{q=1}^{r} \mathrm{e}^{-\mathrm{j} 2 \pi\left(\boldsymbol{\ell}-\boldsymbol{\ell}^{\prime}\right) \mathbf{x}_{q}}
$$

and $\boldsymbol{\ell}, \boldsymbol{\ell}^{\prime} \in[-M, \ldots, M]^{d}$. That is, the matrix $\mathbf{T}_{d}$ is composed of $(2 M+1)^{2}$ blocks $\mathbf{B}_{i}$ of size $(2 M+1)^{d-1} \times(2 M+1)^{d-1}$, each including $(2 M+1)^{2}$ blocks of size $(2 M+1)^{d-2} \times(2 M+$ $1)^{d-2}$, and so on. The smallest blocks have size $(2 M+1) \times$ $(2 M+1)$; they have the same structure as matrix $\mathbf{T}_{1}$ in the unidimensional case; however, only those on the main diagonal have a Hermitian structure. Proof of this is given in [28] for $d=$ 2 ; the extension to the $d$-dimensional case is straightforward.

\section{Estimation ERror CALCUlation Method}

The analysis detailed in the next sections consists of the following main steps.

i) As a practical case, we consider the asymptotic expression of the LMMSE in (11) and notice that an analytical evaluation of the asymptotic LMMSE could be obtained by exploiting the closed-form expression of the eigenvalue distribution, $f_{d, \beta}(x)$, of the reconstruction matrix. However, such expression is still unknown. Hence, as a first step, we derive a closed-form expression of the moments of $\lambda_{d, \beta}$, for any $d$ and $\beta$, and provide an algorithm to compute them.

ii) We show that the value of the moments of the eigenvalue distribution decreases as the field dimension $d$ increases.

iii) We prove that, as $d \rightarrow \infty$, the expression of the eigenvalue distribution tends to the Marčenko-Pastur distribution.

iv) By using the Marčenko-Pastur distribution, we are able to obtain a tight approximation for the LMMSE of the reconstructed field, which holds for any finite value of $d$.

\section{Closed-Form Expression of the Moments of the ASYMPTOTIC EIGENVALUE PDF}

Ideally, we would like to obtain the analytical expression of the distribution $f_{d, \beta}(x)$ of the asymptotic eigenvalue of $\mathbf{T}_{d}$, for a given $\beta$. Unfortunately, such a calculation seems to be prohibitive and is still an open problem. Therefore, as a first step, we compute the closed-form expression of the moments $\mathbb{E}\left[\lambda_{d, \beta}^{p}\right]$ of $\lambda_{d, \beta}$, for any positive integer $p$.
In the limit for $M$ and $r$ growing to infinity with constant $\beta$, the expression of $\mathbb{E}\left[\lambda_{d, \beta}^{p}\right]$ can be easily obtained from the powers of $\mathbf{T}_{d}$ as in [29] and [30]

$$
\mathbb{E}\left[\lambda_{d, \beta}^{p}\right]=\lim _{M, r \rightarrow+\infty} \frac{1}{(2 M+1)^{d}} \operatorname{Tr} \underset{\mathcal{X}}{\mathbb{E}}\left[\mathbf{T}_{d}^{p}\right] .
$$

In Section $\mathrm{V}$-A, we show that $\mathbb{E}\left[\lambda_{d, \beta}^{p}\right]$ is a polynomial in $\beta$, of degree $p-1$ [see (24)]; the remaining subsections describe how to compute this polynomial.

\section{A. Partitions}

Using (12), the term $\operatorname{Tr} \mathbb{E}_{\mathcal{X}}\left[\mathbf{T}_{d}^{p}\right]$ in (13) can be written as

$$
\begin{aligned}
\operatorname{Tr} \underset{\mathcal{X}}{\mathbb{E}}\left[\mathbf{T}_{d}^{p}\right] & =\underset{\mathcal{X}}{\mathbb{E}}\left[\sum_{\boldsymbol{\ell}_{1}}\left(\mathbf{T}_{d}^{p}\right)_{\nu\left(\boldsymbol{\ell}_{1}\right), \nu\left(\boldsymbol{\ell}_{1}\right)}\right] \\
& =\underset{\mathcal{X}}{\mathbb{E}}\left[\sum_{\mathbf{L} \in \mathcal{L}_{d}}\left(\mathbf{T}_{d}\right)_{\nu\left(\boldsymbol{\ell}_{1}\right), \nu\left(\boldsymbol{\ell}_{2}\right)} \cdots\left(\mathbf{T}_{d}\right)_{\nu\left(\boldsymbol{\ell}_{p}\right), \nu\left(\boldsymbol{\ell}_{1}\right)}\right] \\
& =\frac{1}{r^{p}} \sum_{\mathbf{q} \in \mathcal{Q}} \sum_{\mathbf{L} \in \mathcal{L}_{d}} \underset{\mathcal{X}}{\mathbb{E}}\left[\mathrm{e}^{\left.-\mathrm{j} 2 \pi \sum_{i=1}^{p} \mathbf{x}_{q_{i}}^{\mathrm{T}}\left(\boldsymbol{\ell}_{i}-\boldsymbol{\ell}_{[i+1]}\right)\right]}\right.
\end{aligned}
$$

where $\mathcal{Q}=\left\{\mathbf{q} \mid \mathbf{q}=\left[q_{1}, \ldots, q_{p}\right]\right\}, q_{i}=1, \ldots, r \mathcal{L}_{d}=\{\mathbf{L} \mid \mathbf{L}=$ $\left.\left[\ell_{1}, \ldots, \ell_{p}\right]\right\}, \ell_{i}=\left[\ell_{i_{1}}, \cdots, \ell_{i_{d}}\right]^{\mathrm{T}}, \ell_{i_{m}}=-M, \ldots M$ and

$$
[i+1]= \begin{cases}i+1, & 1 \leq i<p \\ 1, & i=p\end{cases}
$$

In (14), the average is performed over the random set of positions $\mathcal{X}=\left\{\mathbf{x}_{1}, \ldots, \mathbf{x}_{r}\right\}$, with independent and uniformly distributed elements. To obtain a closed-form expression of the distribution moments, we rewrite (14) by using set partitioning.

Let $\mathcal{P}=\{1, \ldots, p\}$ be the set of integers from 1 to $p$. We observe that any given vector $\mathbf{q} \in \mathcal{Q}$ partitions, the set $\mathcal{P}$ into $1 \leq k(\mathbf{q}) \leq p$ disjoint nonempty subsets $\mathcal{P}_{1}(\mathbf{q}), \ldots, \mathcal{P}_{k}(\mathbf{q})$, where $\mathcal{P}_{j}, j=1, \ldots, k(\mathbf{q})$, is the set of indexes of the entries of $\mathbf{q}$ taking the same value $\gamma_{j}$. That is,

$$
\mathcal{P}_{j}(\mathbf{q})=\left\{i \in \mathcal{P} \mid q_{i}=\gamma_{j}\right\}
$$

and $k(\mathbf{q})$ is the number of distinct values $\gamma_{j}$ taken by the entries of vector q. Subsets $\mathcal{P}_{j}$ have the following properties:

$$
\bigcup_{j=1}^{k(\mathbf{q})} \mathcal{P}_{j}(\mathbf{q})=\mathcal{P}, \quad \mathcal{P}_{j}(\mathbf{q}) \cap \mathcal{P}_{j^{\prime}}(\mathbf{q})=\emptyset
$$

for $j \neq j^{\prime}$. Also, we point out that, since $r$ is the number of values that the entries $q_{i}$ can take, there exist $r ! /(r-k(\mathbf{q}))$ ! vectors $\mathbf{q} \in \mathcal{Q}$ generating a given partition of $\mathcal{P}$ made of $k(\mathbf{q})$ subsets. In order to clarify the above concepts, we provide an example below.

Example $1::$ Let $p=6$, then $\mathcal{P}=\{1,2,3,4,5,6\}$. Also, let $\mathbf{q}=[4,9,5,5,4,3]$. Since the distinct values in $\mathbf{q}$ are $\gamma_{j}=3$, 


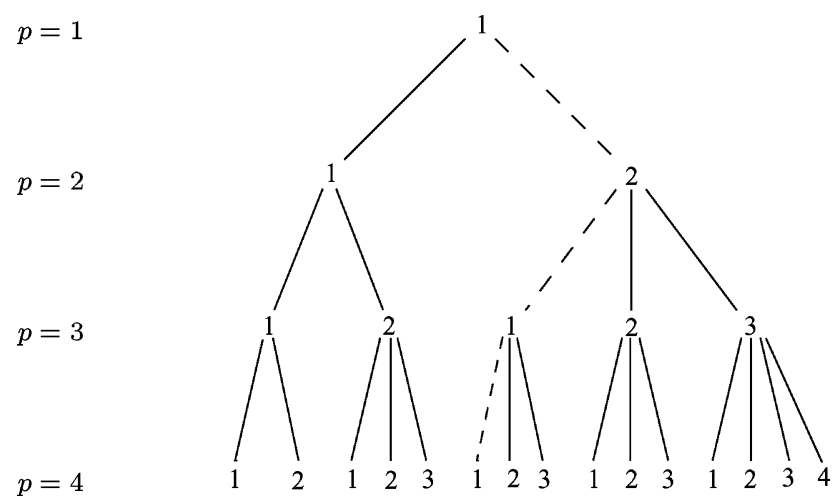

Fig. 1. Partitions tree of depth $p=4$. The path $\boldsymbol{\omega}=[1,2,1,1]$ employed in Example 2 is highlighted by using dashed lines.

$4,5,9$, we have $k(\mathbf{q})=4$. It follows that $\mathcal{P}$ is partitioned into the following subsets:

$$
\begin{aligned}
& \mathcal{P}_{1}(\mathbf{q})=\{1,5\} \quad\left(q_{1}=q_{5}=4\right) \\
& \mathcal{P}_{2}(\mathbf{q})=\{2\} \quad\left(q_{2}=9\right) \\
& \mathcal{P}_{3}(\mathbf{q})=\{3,4\} \quad\left(q_{3}=q_{4}=5\right) \\
& \mathcal{P}_{4}(\mathbf{q})=\{6\} \quad\left(q_{6}=3\right) .
\end{aligned}
$$

Hence, the partition of $\mathcal{P}$ induced by $\mathbf{q}$ is $\{\{1,5\},\{2\},\{3,4\},\{6\}\}$.

Next, we introduce an effective method to represent partitions of a set $\mathcal{P}$, by building a tree of depth $p$, as in Fig. 1. Such a representation will allow to simplify the notation in the following analysis. To build the tree of depth $p$, we proceed as follows. Each node of the tree is assigned with a label from the set $\mathcal{P}=\{1, \ldots, p\}$, starting from the root which is labeled by 1 . Each node generates $m+1$ leaves, labeled in increasing order from 1 to $m+1$, where $m$ is the largest label on the path from the root to such node. Note that, at level $p$, any value in $\{1, \ldots, p\}$ is used to label the leaves at least once.

Then, given a tree of depth $p$, we define $\boldsymbol{\omega}=\left[\omega_{1}, \ldots, \omega_{p}\right]$ as a path of length $p$ from the tree's root to a leaf. We observe that a vector $\mathbf{q}$ can be represented as a path $\boldsymbol{\omega}$ in the tree of depth $p$. This is done by assigning a label $(j=1, \ldots, p)$ in increasing order to every distinct value of $\mathbf{q}$; the vector collecting the labels is the path $\boldsymbol{\omega}$ corresponding to the given $\mathbf{q}$. We have that the path $\boldsymbol{\omega}$, corresponding to a given $\mathbf{q}$, defines in the tree of depth $p$ the same partition of the set $\mathcal{P}$ as the one induced by q. Indeed, given a partition of $\mathcal{P}$, the subset $\mathcal{P}_{j}$ defined in (15) can be rewritten as

$$
\mathcal{P}_{j}(\boldsymbol{\omega})=\left\{i \in \mathcal{P} \mid \omega_{i}=j\right\},
$$

i.e., as the set of integers corresponding to the depths of the $j$ th label in the path. As a last remark, consider the number of distinct values in $\mathbf{q}$ (i.e., the number of distinct labels in $\boldsymbol{\omega}$ ) to be equal to $k(\mathbf{q})$, and recall that the number of all possible values taken by the $p$ elements of $\mathbf{q}$ is equal to $r$. It follows that $r ! /(r-k(\mathbf{q}))$ ! different q's yield the same vector $\boldsymbol{\omega}$. This is in agreement with the fact that, given $\mathcal{P}$, there are $r ! /(r-k(\mathbf{q}))$ ! different q's generating the same partition consisting of $k(\mathbf{q})$ subsets. Again, for the sake of clarity, we give an example.
Example 2: Let us consider $p=4$ and $\mathbf{q}=[4,9,4,4]$. The vector $\mathbf{q}$ can be represented in the tree of depth 4 as the path $\boldsymbol{\omega}=[1,2,1,1]$ (i.e., the path highlighted with dashed lines in Fig. 1). In q there are two distinct values (namely, 4 and 9), or, equivalently, in the path $\boldsymbol{\omega}$ there are two labels (namely, 1 and 2); then $k(\mathbf{q})=k(\boldsymbol{\omega})=2$. Label 1 appears in $\boldsymbol{\omega}$ at depths 1,3 , and $4\left(\mathcal{P}_{1}=\{1,3,4\}\right)$, while label 2 appears at depth $2\left(\mathcal{P}_{2}=\{2\}\right)$. The partition of $\mathcal{P}=\{1,2,3,4\}$ induced by $q$ or, equivalently, by $\boldsymbol{\omega}$ is therefore: $\{\{1,3,4\},\{2\}\}$.

From the discussion above, it should be clear that considering a partition of $\mathcal{P}$ is equivalent to considering a path $\boldsymbol{\omega}$ in a tree of depth $p$. Hence, in the following analysis, we will refer to a partition through its corresponding path $\boldsymbol{\omega}$.

We now exploit set partitioning to rewrite (14). Since the random vectors $\mathbf{x}_{q}$ are independent, given $\mathbf{q}$, the average operator in (14) factorizes into $k(\mathbf{q})$ terms, i.e.,

$$
\begin{aligned}
\underset{\mathcal{X}}{\mathbb{E}}\left[\mathrm{e}^{-\mathrm{j} 2 \pi \sum_{i=1}^{p} \mathbf{x}_{q_{i}}^{\mathrm{T}}\left(\boldsymbol{\ell}_{i}-\boldsymbol{\ell}_{[i+1]}\right)}\right] \\
=\prod_{j=1}^{k(\mathbf{q})} \underset{\mathbf{x}_{\gamma_{j}}}{\mathbb{E}}\left[\mathrm{e}^{-\mathrm{j} 2 \pi \mathbf{x}_{\gamma_{j}}^{\mathrm{T}} \sum_{i \in \mathcal{P}_{j}(\mathbf{q})} \boldsymbol{\ell}_{i}-\boldsymbol{\ell}_{[i+1]}}\right] .
\end{aligned}
$$

Each term depends on a single random vector $\mathbf{x}_{\gamma_{j}}$. Moreover, since the entries of $\mathbf{x}_{\gamma_{j}}$ are independent random variables uniformly distributed in $[0,1)$, we have

$$
\begin{aligned}
\underset{\mathbf{x}_{\gamma_{j}}}{\mathbb{E}}\left[\mathrm{e}^{-\mathrm{j} 2 \pi \mathbf{x}_{\gamma_{j}}^{\mathrm{T}} \sum_{i \in \mathcal{P}_{j}(\mathbf{q})} \boldsymbol{\ell}_{i}-\boldsymbol{\ell}_{[i+1]}}\right] & =\prod_{m=1}^{d} \underset{x_{\gamma_{j}, m}}{\mathbb{E}}\left[\mathrm{e}^{-\mathrm{j} 2 \pi x_{\gamma_{j}, m} c_{j m}}\right] \\
& =\prod_{m=1}^{d} \delta\left(c_{j m}\right)
\end{aligned}
$$

where $x_{\gamma_{j}, m}$ and $\ell_{i, m}$ are the $m$ th entries of $\mathbf{x}_{\gamma_{j}}$ and $\boldsymbol{\ell}_{i}$, respectively, where the function $\delta(\cdot)$ is the Kronecker's delta, and where $c_{j m}=\sum_{i \in \mathcal{P}_{j}(\mathbf{q})} \ell_{i, m}-\ell_{[i+1], m}$. By substituting (17) and (18) in (14) and by expanding the summation $\sum_{\mathbf{L} \in \mathcal{L}_{d}}$, we obtain

$$
\begin{aligned}
\operatorname{Tr} \underset{\mathcal{X}}{\mathbb{E}}\left[\mathbf{T}_{d}^{p}\right] & =\frac{1}{r^{p}} \sum_{\mathbf{q} \in \mathcal{Q}} \sum_{\boldsymbol{\ell}_{1} \in \mathcal{L}_{1}} \ldots \sum_{\boldsymbol{\ell}_{d} \in \mathcal{L}_{1}} \prod_{j=1}^{k(\mathbf{q})} \prod_{m=1}^{d} \delta\left(c_{j m}\right) \\
& =\frac{1}{r^{p}} \sum_{\mathbf{q} \in \mathcal{Q}} \prod_{m=1}^{d}\left[\sum_{\boldsymbol{\ell}_{m} \in \mathcal{L}_{1}} \prod_{j=1}^{k(\mathbf{q})} \delta\left(c_{j m}\right)\right] \\
& =\frac{1}{r^{p}} \sum_{\mathbf{q} \in \mathcal{Q}}\left[\sum_{\boldsymbol{\ell} \in \mathcal{L}_{1}} \prod_{j=1}^{k(\mathbf{q})} \delta\left(c_{j}\right)\right]^{d}
\end{aligned}
$$

where $c_{j}=\sum_{i \in \mathcal{P}_{j}(\mathbf{q})} \ell_{i}-\ell_{[i+1]}$. For any given $\mathbf{q} \in \mathcal{Q}$, the expression

$$
\zeta_{M}(\mathbf{q})=\sum_{\boldsymbol{\ell} \in \mathcal{L}_{1}} \prod_{j=1}^{k(\mathbf{q})} \delta\left(c_{j}\right)
$$

is a polynomial in $M$, since it represents the number of points with integer coordinates contained in the hypercube $[-M, \ldots, M]^{p}$ and satisfying the $k(\mathbf{q})$ constraints

$$
c_{j}=\sum_{i \in \mathcal{P}_{j}(\mathbf{q})} \ell_{i}-\ell_{[i+1]}=0
$$


for $j=1, \ldots, k(\mathbf{q})$. In Appendix A, we show that one of these constraints is always redundant and that the number of linearly independent constraints is exactly equal to $k(\mathbf{q})-1$. As a consequence, the polynomial $\zeta_{M}(\mathbf{q})$ has degree $p-k(\mathbf{q})+1$, and, for large values of $M$, we have $\zeta_{M}(\mathbf{q})=O\left((2 M+1)^{p-k(\mathbf{q})+1}\right)$.

Now, using (19) and (20), the limit in (13) is given by

$$
\mathbb{E}\left[\lambda_{d, \beta}^{p}\right]=\lim _{M, r \rightarrow+\infty} \sum_{\mathbf{q} \in \mathcal{Q}} \frac{\zeta_{M}(\mathbf{q})^{d}}{r^{p}(2 M+1)^{d}} .
$$

Equation (22) can be further simplified by considering that there exist $r ! /(r-k(\mathbf{q}))$ ! vectors $\mathbf{q} \in \mathcal{Q}$ generating a given partition of $\mathcal{P}$ made of $k(\mathbf{q})$ subsets, or, equivalently, a path $\boldsymbol{\omega}$ of length $p$ with $k(\mathbf{q})$ distinct labels.

Let $\Omega_{p}$ be the set of vectors $\boldsymbol{\omega}$, each corresponding to a distinct partition of $\mathcal{P}$. Also, let us write $k(\mathbf{q})$ and $\zeta_{M}(\mathbf{q})$ as functions of the partition induced by $\mathbf{q}$, i.e., as functions of $\boldsymbol{\omega}$. Then, we obtain

$$
\begin{aligned}
\mathbb{E}\left[\lambda_{d, \beta}^{p}\right] & =\lim _{\substack{M, r \rightarrow+\infty \\
\beta}} \sum_{\boldsymbol{\omega} \in \Omega_{p}} \sum_{\mathbf{q} \Rightarrow \boldsymbol{\omega}} \frac{\zeta_{M}(\boldsymbol{\omega})^{d}}{r^{p}(2 M+1)^{d}} \\
& \stackrel{(a)}{=} \lim _{M, r \rightarrow+\infty} \sum_{\boldsymbol{\omega} \in \Omega_{p}} \frac{\zeta_{M}(\boldsymbol{\omega})^{d} r !}{(r-k(\boldsymbol{\omega})) ! r^{p}(2 M+1)^{d}}
\end{aligned}
$$

where the following holds:

- the notation $\sum_{\mathbf{q} \Rightarrow \omega}$ represents the sum over all vectors $\mathbf{q}$ generating a given path $\omega$;

- the equality $(a)$ holds because the number of vectors $\mathbf{q}$ generating a given $\boldsymbol{\omega}$ is $r ! /(r-k(\boldsymbol{\omega})) !$.

Note that, for large $r, r ! /(r-k(\boldsymbol{\omega})) !=r^{k(\boldsymbol{\omega})}+O\left(r^{k(\boldsymbol{\omega})-1}\right)$. Also, since $\zeta_{M}(\boldsymbol{\omega})$ is a polynomial in $M$ of degree $p-k(\boldsymbol{\omega})+1$, for large values of $M$ we have $\zeta_{M}(\boldsymbol{\omega})=$ $v(\boldsymbol{\omega})(2 M+1)^{p-k(\boldsymbol{\omega})+1}+O\left((2 M+1)^{p-k(\boldsymbol{\omega})}\right)$, where $v(\boldsymbol{\omega})$ is the coefficient of degree $p-k(\boldsymbol{\omega})+1$ of $\zeta_{M}(\boldsymbol{\omega})$. Therefore, taking the limit, we obtain

$$
\mathbb{E}\left[\lambda_{d, \beta}^{p}\right]=\sum_{\boldsymbol{\omega} \in \Omega_{p}} v(\boldsymbol{\omega})^{d} \beta^{p-k(\boldsymbol{\omega})}=\sum_{k=1}^{p} \beta^{p-k} \sum_{\boldsymbol{\omega} \in \Omega_{p}, k} v(\boldsymbol{\omega})^{d}
$$

where $\Omega_{p, k} \subseteq \Omega_{p}$ is the subset of $\Omega_{p}$ containing paths with $k(\boldsymbol{\omega})$ distinct labels, and

$$
v(\boldsymbol{\omega})=\lim _{M \rightarrow+\infty} \frac{\zeta_{M}(\boldsymbol{\omega})}{(2 M+1)^{p-k(\boldsymbol{\omega})+1}} .
$$

Note that the coefficient $v(\boldsymbol{\omega})$ represents the volume of the convex polytope described by the constraints in (21), when the variables $\ell_{i}$ are considered as real and limited to a $p$-dimensional hypercube of volume 1 . As a consequence, we have $0 \leq v(\boldsymbol{\omega}) \leq 1$.

Equation (24) provides a closed-form expression of the moment $\mathbb{E}\left[\lambda_{d, \beta}^{p}\right]$, as a polynomial in $\beta$ of degree $p-1$. Again, for the sake of clarity, we give an example below.

Example 3: Let $p=6$ and $\mathbf{q}=[4,9,5,5,4,3]$. We have $\boldsymbol{\omega}=$ $[1,2,3,3,1,4]$, and the partition of $\mathcal{P}$ is $\{\{1,5\},\{2\},\{3,4\},\{6\}\}$. Then, the set of $k(\boldsymbol{\omega})=4$ constraints (as in (21)) are given by

$$
\begin{aligned}
& \ell_{1}+\ell_{5}=\ell_{2}+\ell_{6}, \quad \ell_{2}=\ell_{3}, \\
& \ell_{3}+\ell_{4}=\ell_{4}+\ell_{5}, \quad \ell_{6}=\ell_{1} .
\end{aligned}
$$

The last equation is redundant since can be obtained from the first three constraints. Simplifying, we obtain $\ell_{1}=\ell_{6}$, and $\ell_{2}=\ell_{3}=\ell_{5}$. Since each variable $\ell_{i}$ ranges from $-M$ to $M$, the number of integer solutions satisfying the constraints is exactly $\zeta_{M}(\boldsymbol{\omega})=(2 M+1)^{3}$, and then $v(\boldsymbol{\omega})=\lim _{M \rightarrow+\infty}\left(\zeta_{M}(\boldsymbol{\omega}) /(2 M+1)^{p-k(\boldsymbol{\omega})+1}\right)=1$.

Next, in order to compute $\mathbb{E}\left[\lambda_{d, \beta}^{p}\right]$, we need to following:

- to enumerate the partitions, i.e., the vectors $\boldsymbol{\omega} \in \Omega_{p, k}$, for each $k=1, \ldots, p$ (see Section V-B);

- to compute the coefficients $v(\boldsymbol{\omega})$, for any $\boldsymbol{\omega} \in \Omega_{p, k}$ and $k=1, \ldots, p$ (see Section V-C).

\section{B. Partitions Enumeration}

We notice that $\Omega_{p}$ represents the set of partitions of a $p$-element set, thus it has cardinality $\left|\Omega_{p}\right|=B(p)$, where $B(p)$ is the $p$ th Bell number or exponential number [31]. Furthermore, the subset $\Omega_{p, k} \subseteq \Omega_{p}$ has cardinality $S(p, k)$, which is a Stirling number of the second kind [32] given by

$$
S(p, k)=\frac{1}{k !} \sum_{i=0}^{k}(-1)^{i}\left(\begin{array}{c}
k \\
i
\end{array}\right)(k-i)^{p}
$$

with $B(p)=\sum_{k=1}^{p} S(p, k)$.

\section{Computation of the Coefficients $v(\boldsymbol{\omega})$}

The last step required for the computation of $\mathbb{E}\left[\lambda_{d, \beta}^{p}\right]$ is the evaluation of the coefficients $v(\boldsymbol{\omega})$, for every $\boldsymbol{\omega} \in \Omega_{p}$. We have the following lemma.

Lemma 5.1: For any $\omega \in \Omega_{p}$ (or, equivalently, any partition of $\mathcal{P}$ ) and any arbitrary integer $n$, with $n=1, \ldots, k(\boldsymbol{\omega})$, the coefficient $v(\boldsymbol{\omega})$ in (25) is given by

$$
v(\boldsymbol{\omega})=\left.\int_{\mathbb{R}^{k(\boldsymbol{\omega})-1}} \prod_{i=1}^{p} \operatorname{sinc}\left(y_{\omega_{i}}-y_{\omega_{[i+1]}}\right)\right|_{y_{n}=0} \mathrm{~d} \mathbf{y}_{n}
$$

where:

- $\mathbf{y}_{n}=\left[y_{1}, \ldots, y_{n-1}, y_{n+1}, \ldots, y_{k(\boldsymbol{\omega})}\right]^{\mathrm{T}}$;

- $\operatorname{sinc}(y)=(\sin (\pi y) / \pi y)$.

Proof: The proof can be found in Appendix B.

\section{A Practical Method for the Moments Computation}

Equation (26) in Section $\mathrm{V}-\mathrm{C}$ shows that the computation of $\mathbb{E}\left[\lambda_{d, \beta}^{p}\right]$ requires the evaluation of $B(p)$ integrals. However, $B(p)$ is very large even for small $p$, e.g., $B(10)=115975$ and $B(20) \approx 5 \cdot 10^{13}$.

The computational complexity can be reduced by recursively applying the simplification rules defined in the following lemma.

Lemma 5.2: Let

- $\boldsymbol{\omega}=\left[\omega_{1}, \ldots, \omega_{p}\right]$ be the path in a tree of depth $p$, corresponding to the partition of $\mathcal{P}$ into $k$ subsets;

- $\mathcal{P}_{1}, \ldots, \mathcal{P}_{k}$ be the subsets of $\mathcal{P}=\{1, \ldots, p\}$ defined as in (16);

- $i \in \mathcal{P}_{j}$ :

- $\omega^{\prime}$ be the path obtained from $\omega$ by removing $\omega_{i}$.

We have the following rules:

1) if $\mathcal{P}_{j}$ has cardinality 1 (i.e., $\mathcal{P}_{j}$ is a singleton) or

2) if $\mathcal{P}_{j}$ contains adjacencies (in the circular sense), i.e., both $i$ and $[i+1] \in \mathcal{P}_{j}$,

then $v(\boldsymbol{\omega})=v\left(\boldsymbol{\omega}^{\prime}\right)$. 
TABLE I

EXAMPlE OF COMPLEXITY REDUCTION USING THE RULES DESCRIBED IN LEMMA 5.2

\begin{tabular}{||c||c|c|c||c|c|c||}
\hline \multicolumn{1}{||c||}{} & \multicolumn{3}{c||}{ Example 1 } & \multicolumn{3}{c||}{ Example 2 } \\
\hline Step & $\boldsymbol{\omega}$ & Rule & $i$ & $\boldsymbol{\omega}$ & Rule & $i$ \\
\hline 1 & {$[1,2,3,2,2,1]$} & 1 & 3 & {$[1,2,3,1,2,1]$} & 1 & 3 \\
\hline 2 & {$[1,2,2,2,1]$} & 2 & 2 & {$[1,2,1,2,1]$} & 2 & 5 \\
\hline 3 & {$[1,2,2,1]$} & 2 & 2 & {$[1,2,1,2]$} & & \\
\hline 4 & {$[1,2,1]$} & 1 & 2 & & & \\
\hline 5 & {$[1,1]$} & 2 & 2 & & & \\
\hline 6 & {$[1]$} & 1 & 1 & & & \\
\hline 7 & {[]} & & & \multicolumn{3}{|c||}{$v(\boldsymbol{\omega})=2 / 3$} \\
\hline \multicolumn{6}{|c||}{$v(\boldsymbol{\omega})=1$} & \multicolumn{3}{|c||}{} \\
\hline
\end{tabular}

Proof: The proof is a direct consequence of Lemma 5.1 and can be found in Appendix C.

Table I shows two examples of how the rules described in Lemma 5.1 can be applied. Example 1 in the Table assumes $p=6$ and $\boldsymbol{\omega}=[1,2,3,2,2,1]$. At step 1 , we note that the third element $(i=3)$ of $\boldsymbol{\omega}$ is a singleton, then, by applying rule 1 , we can remove it from the path. At step 2, we find that in $\boldsymbol{\omega}$ there are some adjacencies, hence we apply twice rule 2 (steps 2 and 3). At step 4, the second element of $\boldsymbol{\omega}$ is a singleton, and we remove it by applying rule 1 . Eventually, at step 7 , the path $\boldsymbol{\omega}$ is empty (i.e., has size $p=0$ ) and, thus, the corresponding coefficient is $v(\boldsymbol{\omega})=1\left(\mathbb{E}\left[\lambda_{d, \beta}^{0}\right]=\mathbb{E}[1]=1\right)$.

Example 2 in the table assumes $p=6$ and $\boldsymbol{\omega}=$ $[1,2,3,1,2,1]$. After removing a singleton (step 1) and an adjacency (step 2), the remaining path cannot be further reduced. Then, to compute the coefficient $v(\boldsymbol{\omega})$, we need to apply directly Lemma 5.1 on the path $\boldsymbol{\omega}=[1,2,1,2]$. We obtain

$$
\begin{aligned}
v(\boldsymbol{\omega})= & \int_{-\infty}^{+\infty} \operatorname{sinc}\left(y_{1}-y_{2}\right) \operatorname{sinc}\left(y_{2}-y_{1}\right) \\
& \left.\cdot \operatorname{sinc}\left(y_{1}-y_{2}\right) \operatorname{sinc}\left(y_{2}-y_{1}\right)\right|_{y_{2}=0} \mathrm{~d} y_{1} \\
= & \int_{-\infty}^{+\infty} \operatorname{sinc}\left(y_{1}\right)^{4} \mathrm{~d} y_{1}=\frac{2}{3} .
\end{aligned}
$$

In the following example, Lemmas 5.1 and 5.2 are exploited to explicitly compute $\mathbb{E}\left[\lambda_{d, \beta}^{4}\right] .\{$

Example 4: Let us consider $p=4$. The total number of partitions of $\mathcal{P}=\{1,2,3,4\}$ is equal to $B(4)=15$. Considering the tree of depth $p$, we apply to each path the rules of Lemma 5.2, and we find that 14 paths (partitions) out of 15 reduce to the empty path, thus contributing with $v(\boldsymbol{\omega})=1$. The only path that cannot be further reduced is $\boldsymbol{\omega}=[1,2,1,2]$. Thus, applying Lemma 5.1 with $n=2$, we obtain $v(\boldsymbol{\omega})=2 / 3$. From (24) and considering all contributions, we obtain

$$
\mathbb{E}\left[\lambda_{d, \beta}^{4}\right]=\beta^{3}+\left(6+(2 / 3)^{d}\right) \beta^{2}+6 \beta+1 .
$$

\section{CONVERgence to THE MARČEnKo-PAStuR DISTRIBUTION}

In Section $\mathrm{V}$, we have shown that the moments of the asymptotic eigenvalues of $\mathbf{T}_{d}$ are polynomials in $\beta$, given by (24). In particular, the $p$ th moment $\mathbb{E}\left[\lambda_{d, \beta}^{p}\right]$ has degree $p-1$ and is

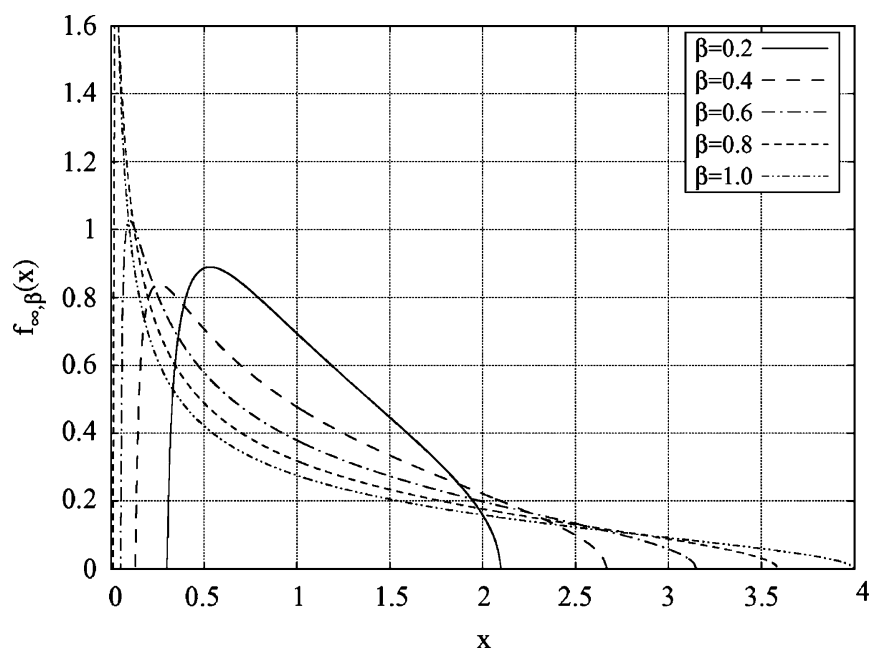

Fig. 2. Marčenko-Pastur distribution.

given by the sum of $B(p)$ positive contributions of the form $v(\boldsymbol{\omega})^{d} \beta^{p-k(\omega)}$. Since $0<v(\boldsymbol{\omega}) \leq 1$ and $\beta>0$, for any $d$, the following inequality holds:

$$
\mathbb{E}\left[\lambda_{d+1, \beta}^{p}\right] \leq \mathbb{E}\left[\lambda_{d, \beta}^{p}\right]
$$

i.e., for any given $p$ and $\beta$, the moments of the asymptotic eigenvalues decrease as the field dimension increases. The series $\mathbb{E}\left[\lambda_{d, \beta}^{p}\right]$, as a function of $d$, is positive and monotonically decreasing, thus it converges to:

$$
\mathbb{E}\left[\lambda_{\infty, \beta}^{p}\right]=\lim _{d \rightarrow+\infty} \mathbb{E}\left[\lambda_{d, \beta}^{p}\right] .
$$

Lemma 6.1: The moments $\mathbb{E}\left[\lambda_{\infty, \beta}^{p}\right]$ are the Narayana polynomials, given by

$$
\mathbb{E}\left[\lambda_{\infty, \beta}^{p}\right]=\sum_{k=1}^{p} T(p, k) \beta^{p-k}
$$

where $T(p, k)=(1 / k)\left(\begin{array}{l}p-1 \\ k-1\end{array}\right)\left(\begin{array}{c}p \\ k-1\end{array}\right)$ are the Narayana numbers [34], [35]. Moreover, the random variable $\lambda_{\infty, \beta}^{p}$ follows the Marčenko-Pastur distribution [6] with pdf (see Fig. 2):

$$
f_{\infty, \beta}(x)=\frac{\sqrt{\left(c_{1}-x\right)\left(x-c_{2}\right)}}{2 \pi x \beta}
$$

where $c_{1}, c_{2}=(1 \pm \sqrt{\beta})^{2}, 0<\beta \leq 1, c_{2} \leq x \leq c_{1}$.

Proof: The proof is given in Appendix D.

In the following, we apply our findings to the study of the LMMSE of a reconstructed multidimensional field; in particular, we exploit the Marčenko-Pastur distribution to compute the expectation in (11).

\section{Study of The ReCONSTRUCTION QuALITY Through THE MARČENKO-PASTUR DISTRIBUTION}

Recall that the MSE provided by the LMMSE filter is [5]

$$
\mathrm{MSE}_{\infty}^{\mathrm{LMMSE}}=\underset{\lambda_{d, \beta}}{\mathbb{E}}\left[\frac{\alpha \beta}{\lambda_{d, \beta}+\alpha \beta}\right]
$$




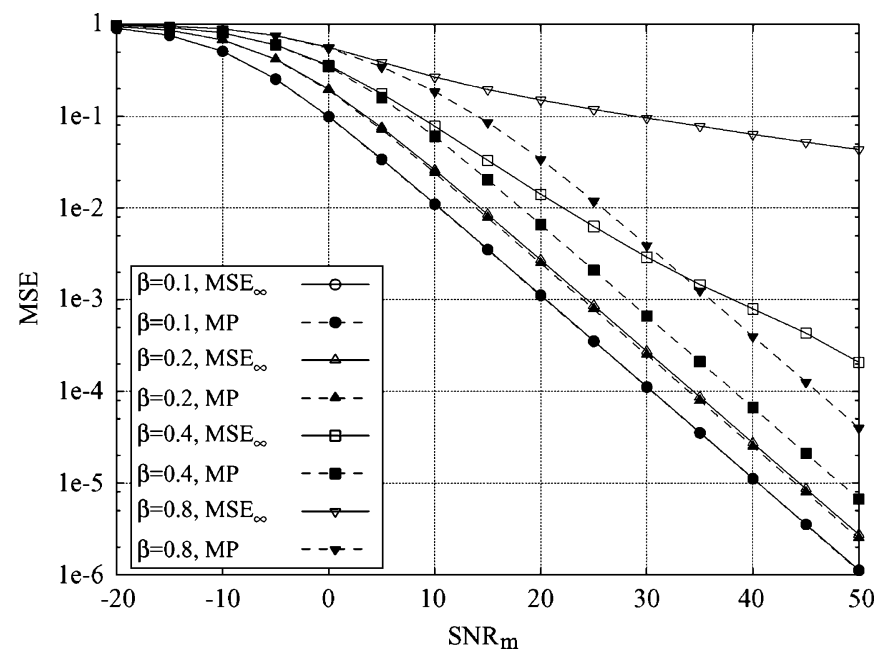

Fig. 3. MSE of the reconstructed field for $d=1$ and varying values of $\beta$. Comparison between the MSE asymptotic value (11) and the fully analytical expression derived using the Marčenko-Pastur distribution (30).

where $\lambda_{d, \beta}$ is distributed as the asymptotic eigenvalues of $\mathbf{T}_{d}$, with pdf $f_{d, \beta}(x)$.

By using the Marčenko-Pastur distribution $f_{\infty, \beta}$ instead of $f_{d, \beta}$, we have

$$
\begin{aligned}
\operatorname{MSE}_{\infty} & =\mathbb{E}_{\lambda_{\infty, \beta}}\left[\frac{\alpha \beta}{\lambda_{\infty, \beta}+\alpha \beta}\right]=\int_{c_{2}}^{c_{1}} \frac{\alpha \beta f_{\infty, \beta}(x)}{x+\alpha \beta} \mathrm{d} x \\
& =\frac{2 \beta-\theta+\sqrt{\theta^{2}-4 \beta}}{2 \beta}
\end{aligned}
$$

where $\theta=1+\beta(1+\alpha)$.

Equation (30) provides an approximation to the $\mathrm{MSE}_{\infty}$, which, as shown in the following plots, can be exploited to derive the quality of the reconstructed field, given a finite $d$.

We first consider $d=1$ and compare in Fig. 3 the expression of the $\mathrm{MSE}_{\infty}$ as in (11) (solid lines) with the one obtained by using the Marčenko-Pastur distribution (dashed lines). The results are presented as functions of the $\mathrm{SNR}_{m}$ and for different values of $\beta$. We computed (11) by averaging over the eigenvalues of 200 realizations of the matrix $\mathbf{T}_{1}$, with $M=150$. The plot shows that, for small values of $\beta$, the Marčenko-Pastur distribution (30) yields an excellent approximation to the $\mathrm{MSE}_{\infty}$, already for $d=1$. Instead, for values of $\beta$ greater than 0.2 , the expression in (30) fails to provide a valid approximation.

However, it is interesting to notice that, for $d>1$, it is possible to obtain an accurate approximation of the $\mathrm{MSE}_{\infty}$ using the Marčenko-Pastur distribution, even for large values of $\beta$. This is shown by Figs. 4 and 5, which plot the results obtained through (11) and (30) for $\beta$ equal to 0.4 and 0.8 , respectively. The results are presented as the $\mathrm{SNR}_{m}$ varies and for different values of the field dimension $d$.

Looking at Fig. 4, we note that our approximation is tight for $d \geq 2$, while Fig. 5 shows that, when $d=3$, we still get a fairly good approximation for $\beta$ as large as 0.8 .

\section{CONCLUSION}

We considered a large-scale wireless sensor network sampling a multidimensional field, and we investigated the mean-

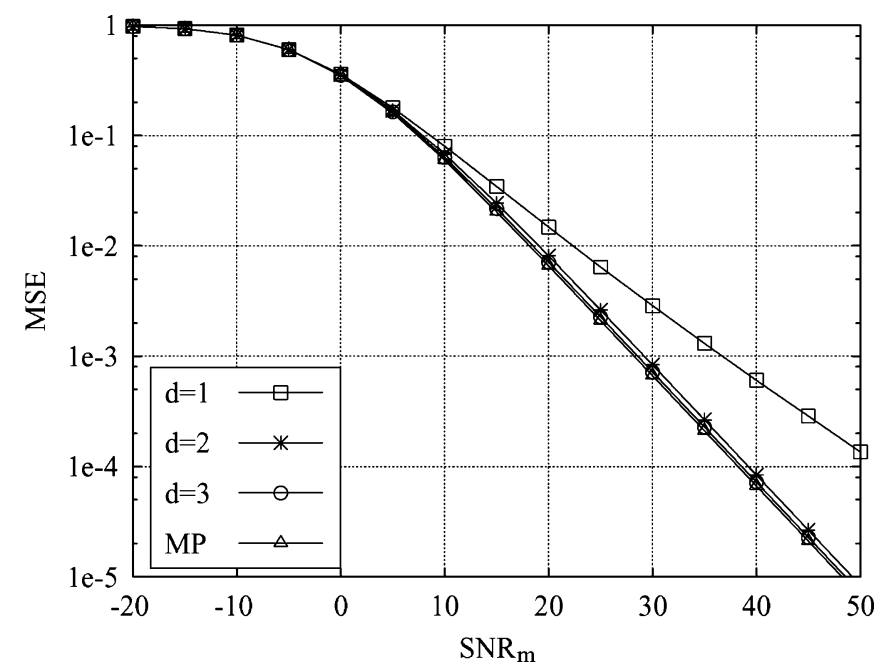

Fig. 4. MSE of the reconstructed field, for $\beta=0.4$ and $d=1,2,3$. Comparison between the MSE asymptotic value (11) and the fully analytical expression derived using the Marčenko-Pastur distribution (30).

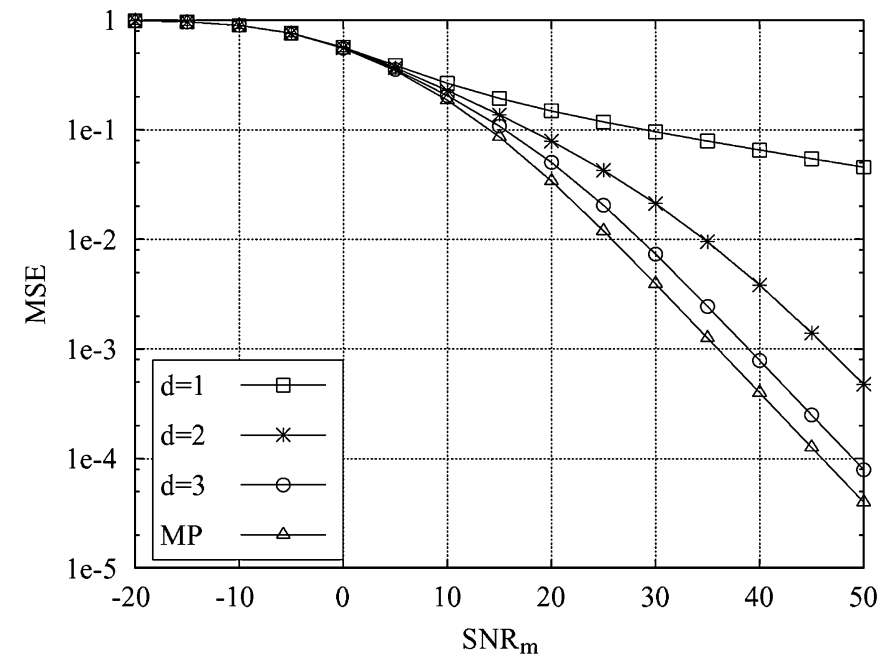

Fig. 5. MSE of the reconstructed field, for $\beta=0.8$ and $d=1,2,3$. Comparison between the MSE asymptotic value (11) and the fully analytical expression derived using the Marčenko-Pastur distribution (30).

square error (MSE) of the signal reconstructed at the sink node. We noticed that an analytical study of the quality of the reconstructed field could be carried out by using the eigenvalue distribution of the matrix representing the sampling system. Since such a distribution is unknown, we first derived a closed-form expression of the distribution moments. By using this expression, we were able to show that the eigenvalue distribution of the reconstruction matrix tends to the Marčenko-Pastur distribution as the field dimension tends to infinity. We applied our results to the study of the MSE of the reconstructed field, when linear filtering is used at the sink node. We found that, by using the Marčenko-Pastur distribution instead of the actual eigenvalue distribution, we obtain a close approximation to the MSE of the reconstructed signal, which holds for field dimensions $d \geq 2$.

We believe that our work is the basis for an analytical study of various aspects concerning the reconstruction quality of multidimensional sensor fields, and, more generally, of irregularly sampled signals. 


\section{APPENDIX A \\ THE CONSTRAINTS}

Let us consider a vector of integers $\mathbf{q}$ of size $p$ partitioning the set $\mathcal{P}=\{1, \ldots, p\}$ in $k$ subsets $\mathcal{P}_{j}, 1 \leq j \leq k$, and the set of $k$ constraints (21). We first show that one of these constraints is always redundant.

\section{A. Redundant Constraint}

Choose an integer $j, 1 \leq j \leq k$. Summing up all constraints except for the $j$ th, we get

$$
\sum_{\substack{h=1 \\ h \neq j}}^{k} c_{h}=\sum_{h=1}^{k} c_{h}-c_{j}=\sum_{i \in \mathcal{P}} \ell_{i}-\ell_{[i+1]}-c_{j}=-c_{j}
$$

which gives the $j$ th constraint since $\sum_{i \in \mathcal{P}} \ell_{i}-\ell_{[i+1]}=0$. Thus, one of the constraints in (21) is always redundant. Next, we show that the remaining $k-1$ constraints are linearly independent.

\section{B. Linear Independence}

The $k$ constraints in (21) can be arranged in the form: $\mathbf{W} \boldsymbol{\ell}=$ $\mathbf{0}$ with $\boldsymbol{\ell}=\left[\ell_{1}, \ldots, \ell_{p}\right]^{\mathrm{T}}$ and $\mathbf{W}$ being a $k \times p$ matrix defined as

$$
\mathbf{W}=\mathbf{W}^{\prime}-\mathbf{W}^{\prime \prime}
$$

where

$$
\left(\mathbf{W}^{\prime}\right)_{j, i}= \begin{cases}+1, & i \in \mathcal{P}_{j} \\ 0, & \text { otherwise }\end{cases}
$$

and $\mathbf{W}^{\prime \prime}$ is obtained from $\mathbf{W}^{\prime}$ by circularly shifting the rows to the right by one position. Since one of the constraints (21) is redundant, the rank of $\mathbf{W}$ is $\rho(\mathbf{W}) \leq k-1$. Now we prove that the rank of $\mathbf{W}$ is equal to $k-1$.

Since the subsets $\mathcal{P}_{j}$ have empty intersection, the rows of $\mathbf{W}^{\prime}$ are linearly independent; hence, $\mathbf{W}^{\prime}$ has rank $k$. Also, $\mathbf{W}^{\prime \prime}$ is obtained from $\mathbf{W}^{\prime}$ by circularly shifting the rows by one position to the right, thus $\mathbf{W}^{\prime \prime}$ can be written as $\mathbf{W}^{\prime \prime}=\mathbf{W}^{\prime} \mathbf{S}$ where $\mathbf{S}$ is the $p \times p$ right-shift matrix [36], i.e., the entries of the $i$ th row of $\mathbf{S}$ are zeros except for an entry equal to 1 at position $[i+1]$. As a consequence, $\mathbf{W}=\mathbf{W}^{\prime}-\mathbf{W}^{\prime} \mathbf{S}=\mathbf{W}^{\prime}\left(\mathbf{I}_{p}-\mathbf{S}\right)$ where the rows of the matrix $\mathbf{I}_{p}-\mathbf{S}$ are obtained by circularly shifting the vector $[+1,-1,0, \ldots, 0]$ and thus has rank $\rho\left(\mathbf{I}_{p}-\mathbf{S}\right)=p-1$. Hence, using the properties of the rank of matrix products reported in [36], we have

$\rho(\mathbf{W})=\rho\left(\mathbf{W}^{\prime}\left(\mathbf{I}_{p}-\mathbf{S}\right)\right) \geq \rho\left(\mathbf{W}^{\prime}\right)+\rho\left(\mathbf{I}_{p}-\mathbf{S}\right)-p=k-1$.

We recall that the system of linear equations $\mathbf{W z}$ has a finite number of integer solutions bounded in $[-M, \ldots, M]^{d}$. The number of solutions decreases as $\rho(\mathbf{W})$ increases.

\section{APPENDIX B}

PROOF OF LEMMA 5.1

Proof: Using (20) and (25), we obtain

$$
v(\boldsymbol{\omega})=\lim _{M \rightarrow+\infty} \frac{1}{(2 M+1)^{p-k(\boldsymbol{\omega})+1}} \sum_{\boldsymbol{\ell} \in \mathcal{L}_{1}} \prod_{j=1}^{k(\boldsymbol{\omega})} \delta\left(c_{j}\right) .
$$

We first notice that $\prod_{j=1}^{k(\boldsymbol{\omega})} \delta\left(c_{j}\right)=\delta(\mathbf{W} \boldsymbol{\ell})$ where the $k(\boldsymbol{\omega}) \times p$ matrix $\mathbf{W}$ is defined in Appendix $\mathrm{A}$ and $\delta(\mathbf{W} \boldsymbol{\ell})$ is a multidimensional Kronecker delta [33]. Since the rank of $\mathbf{W}$ is $\rho(\mathbf{W})=$ $k(\boldsymbol{\omega})-1$ (see Appendix A), then $\delta(\mathbf{W} \boldsymbol{\ell})$ defines a subspace of $\mathbb{Z}^{p}$ with $p-k(\boldsymbol{\omega})+1$ dimensions. Therefore, considering that $\boldsymbol{\ell}$ is a vector of integers with entries ranging in the interval $[-M, \ldots, M]$ and taking the limit for $M \rightarrow \infty$, we obtain $v(\boldsymbol{\omega})=\int_{[-1 / 2,1 / 2)^{p}} \delta_{d}(\mathbf{W} \mathbf{z}) \mathrm{d} \mathbf{z}$ where $\mathbf{z} \in \mathbb{R}^{p}$ and the function $\delta_{d}$ represents the Dirac delta. We have that $\delta_{d}(\mathbf{W z})$ can be factorized as

$$
\delta_{d}(\mathbf{W} \mathbf{z})=\prod_{j=1}^{k(\boldsymbol{\omega})} \delta_{d}\left(\mathbf{r}_{j}^{\mathrm{T}} \mathbf{z}\right)
$$

where $\mathbf{r}_{j}^{\mathrm{T}}$ is the $j$ th row of $\mathbf{W}$. As already shown in Appendix A, one of the constraints (21) is redundant and, hence, one of the factors in the right-hand side of (32), say the $n$ th, must not be included in the product. Now, moving to the Fourier transform domain, we can write $\delta_{d}\left(\mathbf{r}_{j}^{\mathrm{T}} \mathbf{z}\right)=\int_{-\infty}^{+\infty} \exp \left(\mathrm{j} 2 \pi y \mathbf{r}_{j}^{\mathrm{T}} \mathbf{z}\right) \mathrm{d} y$. Therefore

$$
\begin{aligned}
v(\boldsymbol{\omega}) & =\int_{[-1 / 2,1 / 2)^{p}} \prod_{j=1, j \neq n}^{k(\boldsymbol{\omega})} \delta_{d}\left(\mathbf{r}_{j}^{\mathrm{T}} \mathbf{z}\right) \mathrm{d} \mathbf{z} \\
& =\int_{[-1 / 2,1 / 2)^{p}} \prod_{j=1, j \neq n_{-\infty}}^{k(\boldsymbol{\omega})} \int_{[-1 / 2,1 / 2)^{p}}^{+\infty} \mathrm{e}^{\mathrm{j} 2 \pi y_{j} \mathbf{r}_{j}^{\mathrm{T}} \mathbf{z}^{k-1}} \mathrm{~d} y_{j} \mathrm{~d} \mathbf{z} \\
& =\int^{\mathrm{j} 2 \pi \sum_{j=1, j \neq n}^{k(\boldsymbol{\omega})} y_{j} \mathbf{r}_{j}^{\mathrm{T}} \mathbf{z}} \mathrm{d} \mathbf{y}_{n} \mathrm{~d} \mathbf{z}
\end{aligned}
$$

where $\mathbf{y}_{n}=\left[y_{1}, \ldots, y_{n-1}, y_{n+1}, \ldots, y_{k(\boldsymbol{\omega})}\right]^{\mathrm{T}}$. Integrating first with respect to $\mathbf{z}$, we get

$$
\begin{aligned}
v(\boldsymbol{\omega}) & =\int_{\mathbb{R}^{k-1}} \int_{[-1 / 2,1 / 2)^{p}} \mathrm{e}^{\mathrm{j} 2 \pi \sum_{i=1}^{p} z_{i} \mathbf{y}_{n}^{\mathrm{T}} \mathbf{w}_{i}} \mathrm{~d} \mathbf{z} \mathrm{d} \mathbf{y}_{n} \\
& =\int_{\mathbb{R}^{k-1}} \int_{[-1 / 2,1 / 2)^{p}} \prod_{i=1}^{p} \mathrm{e}^{\mathrm{j} 2 \pi z_{i} \mathbf{y}_{n}^{\mathrm{T}} \mathbf{w}_{i}} \mathrm{~d} \mathbf{z} \mathrm{d} \mathbf{y}_{n} \\
& =\int_{\mathbb{R}^{k-1}} \prod_{i=1}^{p} \int_{-1 / 2}^{1 / 2} \mathrm{e}^{\mathrm{j} 2 \pi z_{i} \mathbf{y}_{n}^{\mathrm{T}} \mathbf{w}_{i}} \mathrm{~d} z_{i} \mathrm{~d} \mathbf{y}_{n} \\
& =\int_{\mathbb{R}^{k-1}} \prod_{i=1}^{p} \mathrm{e}^{\mathrm{j} \pi \mathbf{y}_{n}^{\mathrm{T}} \mathbf{w}_{i}} \operatorname{sinc}\left(\mathbf{y}_{n}^{\mathrm{T}} \mathbf{w}_{i}\right) \mathrm{d} \mathbf{y}_{n} \\
& =\int_{\mathbb{R}^{k-1}} \mathrm{e}^{\mathrm{j} \pi \mathbf{y}_{n}^{\mathrm{T}} \sum_{i=1}^{p} \mathbf{w}_{i}} \prod_{i=1}^{p} \operatorname{sinc}\left(\mathbf{y}_{n}^{\mathrm{T}} \mathbf{w}_{i}\right) \mathrm{d} \mathbf{y}_{n}
\end{aligned}
$$

where $\mathbf{w}_{i}$ is the $i$ th column of $\mathbf{W}$, taken after removing its $n$th row. By definition, the $j$ th rows of $\mathbf{W}^{\prime}$ and of $\mathbf{W}^{\prime \prime}$ contain both $p-\left|\mathcal{P}_{j}\right|$ "0" and $\left|\mathcal{P}_{j}\right|$ " +1 ". Since $\mathbf{W}=\mathbf{W}^{\prime}-\mathbf{W}^{\prime \prime}$, we have $\sum_{i=1}^{p} \mathbf{w}_{i}=\mathbf{0}$ and $v(\boldsymbol{\omega})=\int_{\mathbb{R}^{k-1}} \prod_{i=1}^{p} \operatorname{sinc}\left(\mathbf{y}_{n}^{\mathrm{T}} \mathbf{w}_{i}\right) \mathrm{d} \mathbf{y}_{n}$. Notice that, by definition of $\mathbf{W}(\operatorname{see}(31)), \mathbf{y}_{n}^{\mathrm{T}} \mathbf{w}_{i}=y_{j}-\left.y_{j^{\prime}}\right|_{y_{n}=0}$ 
if $i \in \mathcal{P}_{j}$ and $[i+1] \in \mathcal{P}_{j^{\prime}}$. Moreover, by the definition in (16), we have $y_{j}=y_{\omega_{i}}$ when $i \in \mathcal{P}_{j}$. Thus

$$
v(\boldsymbol{\omega})=\left.\int_{\mathbb{R}^{k-1}} \prod_{i=1}^{p} \operatorname{sinc}\left(y_{\omega_{i}}-y_{\omega_{[i+1]}}\right)\right|_{y_{n}=0} \mathrm{~d} \mathbf{y}_{n} .
$$

\section{APPENDIX C}

\section{PROOF OF LEMMA 5.2 (SIMPLIFICATION RULES)}

Let $\mathcal{P}_{j}$ be a singleton with $\mathcal{P}_{j}=i$ and $\omega_{i}=j$. We first notice that, since $\mathcal{P}_{j}$ is a singleton, $[i-1],[i+1] \notin \mathcal{P}_{j}$. By applying Lemma 5.1 with an arbitrary $n \neq j$, we have

$$
\begin{aligned}
v(\boldsymbol{\omega})= & \left.\int_{\mathbb{R}^{k-1}} \prod_{h=1}^{p} \operatorname{sinc}\left(y_{\omega_{h}}-y_{\omega_{[h+1]}}\right)\right|_{y_{n}=0} \mathrm{~d} \mathbf{y}_{n} \\
= & \int_{\substack{\left.\mathbb{R}^{k-1} \\
\text { c. } \\
h \neq 1 \neq i-1\right] \\
h \neq 1}}^{p} \operatorname{sinc}\left(y_{\omega_{h}}-y_{\omega_{[h+1]}}\right) \operatorname{sinc}\left(y_{\omega_{[i-1]}}-y_{\omega_{i}}\right) \\
& \left.\cdot \operatorname{sinc}\left(y_{\omega_{i}}-y_{\omega_{[i+1]}}\right)\right|_{y_{n}=0} \mathrm{~d} \mathbf{y}_{n} .
\end{aligned}
$$

We now integrate with respect to $y_{j}$, with $j=\omega_{i}$ and we obtain

$$
\begin{aligned}
v(\boldsymbol{\omega})= & \int_{\mathbb{R}^{k-2}} \prod_{\substack{h=1 \\
h \neq i \\
h \neq i-1]}}^{p} \operatorname{sinc}\left(y_{\omega_{h}}-y_{\omega_{[h+1]}}\right) \\
& \left.\cdot \int_{\mathbb{R}} \operatorname{sinc}\left(y_{\omega_{[i-1]}}-y_{j}\right) \operatorname{sinc}\left(y_{j}-y_{\omega_{[i+1]}}\right)\right|_{y_{n}=0} \mathrm{~d} y_{j} \mathrm{~d} \mathbf{y}_{n}^{\prime} \\
= & \int_{\mathbb{R}^{k-2}} \prod_{\substack{h=1 \\
h \neq 1 \\
h \neq[i-1]}}^{p} \operatorname{sinc}\left(y_{\omega_{h}}-y_{\omega_{[h+1]}}\right) \\
& \left.\cdot \operatorname{sinc}\left(y_{\omega_{[i-1]}}-y_{\omega_{[i+1]}}\right)\right|_{y_{n}=0} \mathrm{~d} \mathbf{y}_{n}^{\prime} \\
= & \left.\int_{\mathbb{R}^{k-2}}^{\substack{p-1 \\
h=1}} \operatorname{sinc}\left(y_{\omega_{h}^{\prime}}-y_{\omega_{[h+1]}^{\prime}}\right)\right|_{y_{n}=0} \mathrm{~d} \mathbf{y}_{n}^{\prime}=v\left(\boldsymbol{\omega}^{\prime}\right)
\end{aligned}
$$

where $\mathbf{y}_{n}^{\prime}$ and $\boldsymbol{\omega}^{\prime}$ have been obtained from $\mathbf{y}_{n}$ and $\boldsymbol{\omega}$ by removing their $j$ th and $i$ th element, respectively. Obviously, $\mathbf{y}_{n}^{\prime}$ has size $k-1$ and $\boldsymbol{\omega}^{\prime}$ has size $p-1$. Let $\mathcal{P}_{j}$ be such that $\mathcal{P}_{j}=i,[i+1]$, i.e., $\omega_{i}=\omega_{[i+1]}=j$. Then

$$
\begin{aligned}
v(\boldsymbol{\omega})= & \left.\int_{\mathbb{R}^{k-1}} \prod_{h=1}^{p} \operatorname{sinc}\left(y_{\omega_{h}}-y_{\omega_{[h+1]}}\right)\right|_{y_{n}=0} \mathrm{~d} \mathbf{y}_{n} \\
= & \int_{\mathbb{R}^{k-1}} \prod_{\substack{h=1 \\
h \neq i}}^{p} \operatorname{sinc}\left(y_{\omega_{h}}-y_{\omega_{[h+1]}}\right) \\
& \left.\cdot \operatorname{sinc}\left(y_{\omega_{i}}-y_{\omega_{[i+1]}}\right)\right|_{y_{n}=0} \mathrm{~d} \mathbf{y}_{n} \\
= & \left.\int_{\mathbb{R}^{k-1}} \prod_{\substack{h=1 \\
h \neq i}}^{p} \operatorname{sinc}\left(y_{\omega_{h}}-y_{\omega_{[h+1]}}\right) \operatorname{sinc}\left(y_{j}-y_{j}\right)\right|_{y_{n}=0} \mathrm{~d} \mathbf{y}_{n} \\
= & \left.\int_{\mathbb{R}^{k-1}} \prod_{\substack{h=1 \\
h \neq i}}^{p} \operatorname{sinc}\left(y_{\omega_{h}}-y_{\omega_{[h+1]}}\right)\right|_{y_{n}=0} \mathrm{~d} \mathbf{y}_{n} \\
= & \left.\int_{\mathbb{R}^{k-1}}^{p-1} \prod_{h=1}^{p-1} \operatorname{sinc}\left(y_{\omega_{h}^{\prime}}-y_{\omega_{[h+1]}^{\prime}}\right)\right|_{y_{n}=0} \mathrm{~d} \mathbf{y}_{n}=v\left(\boldsymbol{\omega}^{\prime}\right)
\end{aligned}
$$

where $\boldsymbol{\omega}^{\prime}$ has been obtained from $\boldsymbol{\omega}$ by removing its $i$ th element. APPENDIX D

\section{ProOf OF LEMMA 6.1}

In order to prove Lemma 6.1, we first note that $\Omega_{p, k}$ may contain both crossing and noncrossing partitions [37].

a) Noncrossing partitions: Every noncrossing partition contains at least a singleton or a subset with adjacencies, and therefore can be reduced by using the rules in Lemma 5.2. After reduction, the resulting partition is still noncrossing, thus it can be further reduced till the empty set is reached. It follows that the noncrossing partition $\boldsymbol{\omega} \in \Omega_{p, k}$ contributes to the expression of $\mathbb{E}\left[\lambda_{p, k}^{p}\right]$ with a coefficient $v(\boldsymbol{\omega})=1$.

b) Crossing partitions: Recall that, in general, the coefficient $v(\boldsymbol{\omega})$ defined in (26) can be obtained by counting the solutions of the system of equations: $\mathbf{W} \boldsymbol{\ell}=\mathbf{0}$ where the $k(\boldsymbol{\omega}) \times p$ matrix $\mathbf{W}$ contains the coefficients of the $k(\boldsymbol{\omega})$ constraints in (21).

If $\boldsymbol{\omega} \in \Omega_{p, k}$ is a crossing partition, then:

- $k(\boldsymbol{\omega}) \geq 2$ (by definition, a partition with $k(\boldsymbol{\omega})=1$ is always noncrossing);

- it contains at least two subsets $\mathcal{P}_{j}$ and $\mathcal{P}_{j^{\prime}}$, with $j \neq j^{\prime}$, which are crossing.

Some crossing partitions can be reduced by applying the rules in Lemma 5.2 but, even after reduction, they remain crossing.

Let us now focus on the crossing subset $\mathcal{P}_{j}$ of a partition $\boldsymbol{\omega}$ which has been reduced by applying the rules in Lemma 5.2. Without loss of generality, we assume that $\left|\mathcal{P}_{j}\right|=h$, i.e., the partition $\mathcal{P}_{j}$ contains $h$ elements with $h \geq 2$ since $\mathcal{P}_{j}$ is not a singleton. Then, by definition of the matrix $\mathbf{W}$ (see Appendix A) its $j$ th row, $\mathbf{r}_{j}^{\mathrm{T}}$, contains $h$ entries with value 1 , $h$ entries with value -1 and $p-2 h$ zeros. We then build the $2 \times p$ matrix $\widetilde{\mathbf{W}}$ as $\widetilde{\mathbf{W}}=\left[\mathbf{r}_{j},-\mathbf{r}_{j}\right]^{\mathrm{T}}$. Notice that $\widetilde{\mathbf{W}}$ has rank 1 and the system of equations $\widetilde{\mathbf{W}} \boldsymbol{\ell}=\mathbf{0}$ contains the constraints induced by a partition $\widetilde{\boldsymbol{\omega}}=[1,2, \ldots, 1,2]$ with $2 h$ entries. Since the system of equations $\widetilde{\mathbf{W}} \boldsymbol{\ell}=\mathbf{0}$ contains a reduced set of constraints with respect to $\mathbf{W} \boldsymbol{\ell}=\mathbf{0}$ and, thus, a larger number of solutions, it follows that $v(\boldsymbol{\omega}) \leq v(\widetilde{\boldsymbol{\omega}})$.

It is straightforward to show that for a partition such as $\widetilde{\boldsymbol{\omega}}$, with $k(\widetilde{\boldsymbol{\omega}})=2$, the coefficient $v(\widetilde{\boldsymbol{\omega}})$ is given by Lemma 5.1 as $v(\widetilde{\boldsymbol{\omega}})=\int_{\mathbb{R}} \operatorname{sinc}(y)^{2 h} \mathrm{~d} y$. This is a decreasing function of $h$ and since $h \geq 2$ we have

$$
v(\widetilde{\boldsymbol{\omega}})=\int_{\mathbb{R}} \operatorname{sinc}(y)^{2 h} \mathrm{~d} y \leq \int_{\mathbb{R}} \operatorname{sinc}(y)^{4} \mathrm{~d} y=\frac{2}{3} .
$$

Therefore, we conclude that $v(\boldsymbol{\omega}) \leq v(\widetilde{\boldsymbol{\omega}})<1$.

c) Crossing and noncrossing partitions: Let $\Omega_{p, k}^{c}, \Omega_{p, k}^{n} \subset$ $\Omega_{p, k}$ be, respectively, the set of crossing and noncrossing partitions of $\Omega_{p, k}$, with $\Omega_{p, k}^{c} \cap \Omega_{p, k}^{n}=\emptyset$ and $\Omega_{p, k}^{c} \cup \Omega_{p, k}^{n}=\Omega_{p, k}$.

$$
\begin{aligned}
\underset{\mathbb{E}}{\mathbb{E}\left[\lambda_{\infty, \beta}^{p}\right]} & =\lim _{d \rightarrow+\infty} \sum_{k=1}^{p} \beta^{p-k} \sum_{\boldsymbol{\omega} \in \Omega_{p, k}} v(\boldsymbol{\omega})^{d} \\
& =\lim _{d \rightarrow+\infty} \sum_{k=1}^{p} \beta^{p-k}\left(\sum_{\boldsymbol{\omega} \in \Omega_{p, k}^{c}} v(\boldsymbol{\omega})^{d}+\sum_{\boldsymbol{\omega} \in \Omega_{p, k}^{n}} v(\boldsymbol{\omega})^{d}\right) \\
& \stackrel{(a)}{=} \sum_{k=1}^{p} \beta^{p-k}\left|\Omega_{p, k}^{n}\right|
\end{aligned}
$$


where the equality $(a)$ is due to the fact that for noncrossing partitions $v(\boldsymbol{\omega})=1$, while for crossing partitions $v(\boldsymbol{\omega})<1$ and, hence, $\lim _{d \rightarrow+\infty} v(\boldsymbol{\omega})^{d}=0$. In [38], it can be found that the number of noncrossing partitions of size $k$ in a $p$-element set is given by the Narayana numbers $T(p, k)=\left|\Omega_{p, k}^{n}\right|$ and therefore $\mathbb{E}\left[\lambda_{\infty, \beta}^{p}\right]=\sum_{k=1}^{p} T(p, k) \beta^{p-k}$ are the Narayana polynomials. In [6], it is shown that the Narayana polynomials are the moments of the Marčenko-Pastur distribution.

\section{APPENDIX E \\ PROOF OF (11)}

We show that when the LMMSE filter is used, the expression of the asymptotic MSE is given by (11). Indeed, by using (10), (7), (8), and (9) we have

$$
\mathrm{MSE}_{\infty}=\lim _{M, r \rightarrow+\infty} \frac{1}{\sigma_{a}^{2}(2 M+1)^{d}} \underset{\mathcal{X}}{\mathbb{E}} \underset{\mathbf{a}, \mathbf{n}}{\mathbb{E}}\left[\left\|\mathbf{A}_{d}^{-1} \mathbf{G}_{d} \mathbf{p}-\mathbf{a}\right\|^{2}\right]
$$

where $\mathbf{A}_{d}=\mathbf{R}_{d}+\alpha \mathbf{I}$ and $\mathbf{R}_{d}=\mathbf{G}_{d} \mathbf{G}_{d}^{\dagger}$. Substituting (4) in the above expression and assuming $\mathbb{E}\left[\mathbf{a a}^{\dagger}\right]=\sigma_{a}^{2} \mathbf{I}$ and $\mathbb{E}\left[\mathbf{n n}^{\dagger}\right]=$ $\sigma_{n}^{2} \mathbf{I}$, we get

$$
\begin{aligned}
\frac{1}{\sigma_{a}^{2}} \underset{\mathbf{a}, \mathbf{n}}{\mathbb{E}}\left[\left\|\mathbf{A}_{d}^{-1} \mathbf{G}_{d} \mathbf{p}-\mathbf{a}\right\|^{2}\right] \\
\quad=\operatorname{Tr}\left\{\left(\mathbf{A}_{d}^{-1} \mathbf{R}_{d}-\mathbf{I}\right)\left(\mathbf{A}^{-1} \mathbf{R}_{d}-\mathbf{I}\right)^{\dagger}+\alpha \mathbf{A}_{d}^{-1} \mathbf{R}_{d} \mathbf{A}_{d}^{-1}\right\} \\
\quad=\operatorname{Tr}\left\{\alpha\left(\mathbf{R}_{d}+\alpha \mathbf{I}\right)^{-1}\right\}=\operatorname{Tr}\left\{\alpha \beta\left(\mathbf{T}_{d}+\alpha \beta \mathbf{I}\right)^{-1}\right\}
\end{aligned}
$$

where $\mathbf{T}_{d}=\beta \mathbf{R}_{d}$. Let us consider an analytic function $g(\cdot)$ in $\mathbb{R}^{+}$. Let $\mathbf{X}=\mathbf{U} \boldsymbol{\Lambda} \mathbf{U}^{\dagger}$ be a random positive definite Hermitian $n \times n$ matrix, where $\mathbf{U}$ is the eigenvectors matrix of $\mathbf{X}$ and $\Lambda$ is a diagonal matrix containing the eigenvalues of $\mathbf{X}$. By using the result for symmetric matrices in [39, Ch. 6] combined with the result in [40, p. 481], we have $\lim _{n \rightarrow \infty}(1 / n) \operatorname{Tr} \mathbb{E}[g(\mathbf{X})]=\mathbb{E}_{\lambda}[g(\lambda)]$ where the random variable $\lambda$ is distributed as the asymptotic eigenvalues of $\mathbf{X}$. It follows that

$$
\lim _{M, r \rightarrow+\infty} \frac{\operatorname{Tr} \underset{\mathcal{X}}{\mathbb{E}}\left[\alpha \beta\left(\mathbf{T}_{d}+\alpha \beta \mathbf{I}\right)^{-1}\right]}{(2 M+1)^{d}}=\underset{\lambda_{\beta, d}}{\mathbb{E}}\left[\frac{\alpha \beta}{\lambda_{\beta, d}+\alpha \beta}\right] .
$$

\section{REFERENCES}

[1] D. Moore, J. Leonard, D. Rus, and S. Teller, "Robust distributed network localization with noisy range measurements," in Proc. ACM Conf. Embedded Networked Sensor Systems (SenSys 2004), Baltimore, MD, Nov. 2004, pp. 50-61.

[2] H. G. Feichtinger and K. Grchenig, "Theory and practice of irregular sampling," in Wavelets: Mathematics and Applications, J. J. Benedetto and M. W. Frazier, Eds. Boca Raton, FL: CRC Press, 1994, pp. 305-363.

[3] H. G. Feichtinger, K. Gröchenig, and T. Strohmer, "Efficient numerical methods in non-uniform sampling theory," Numeri. Math., vol. 69, pp. 423-440, 1995.

[4] C.-F. Chiasserini, A. Nordio, and E. Viterbo, "On data acquisition and field reconstruction in wireless sensor networks," in Signals and Communication Technology, Distributed Cooperative Laboratories: Networking, Instrumentation, and Measurements, F. Davoli, S. Palazzo, and S. Zappatore, Eds. Berlin Heidelberg: Springer, 2006, pp. $161-171$.

[5] A. Nordio, C.-F. Chiasserini, and E. Viterbo, "Quality of field reconstruction in sensor networks," in Proc. IEEE INFOCOM Mini-Symposium, Anchorage, AK, May 2007.

[6] V. A. Marčenko and L. A. Pastur, "Distribution of eigenvalues for some sets of random matrices," USSR Sbornik, vol. 1, pp. 457-483, 1967.
[7] P. Stoica and R. Moses, Introduction to Spectral Analysis. Upper Saddle River, NJ: Prentice-Hall, 2000.

[8] F. A. Marvasti, Nonuniform Sampling: Theory and Practice. New York: Kluwer Academic: Plenum, 2001.

[9] H. Rauhut, "Random sampling of sparse trigonometric polynomials," [Online]. Available: http://homepage.univie.ac.at/holger.rauhut/RandomSampling.pdf

[10] H. G. Feichtinger and K. Gröchenig, "Error analysis in regular and irregular sampling theory," Appl. Anal., vol. 50, pp. 167-189, 1993.

[11] H. Rauhut, "Stability results for random sampling of sparse trigonometric polynomials," [Online]. Available: http://homepage.univie.ac.at/holger.rauhut/StabilityRS.pdf

[12] E. Candes and T. Tao, "The Dantzig selector: statistical estimation when $p$ is much larger than $n$," [Online]. Available: http://www.acm. caltech.edu/ emmanuel/papers/DantzigSelector.pdf

[13] A. Aldroubi and K. Gröchenig, "Nonuniform sampling and reconstruction in shift-invariant spaces," SIAM Rev., vol. 43, pp. 585-620, Dec. 2001.

[14] K. Gröchenig, "Irregular sampling, Toeplitz matrices, and the approximation of entire functions of exponential type," Math. Comput., vol. 68, no. 226, pp. 749-765, Apr. 1999.

[15] M. Perillo, Z. Ignjatovic, and W. Heinzelman, "An energy conservation method for wireless sensor networks employing a blue noise spatial sampling technique," in Proc. Int. Conf. Information Processing in Sensor Networks (IPSN), Berkeley, CA, Apr. 2004

[16] R. Willett, A. Martin, and R. Nowak, "Backcasting: Adaptive sampling for sensor networks," in Proc. Int. Conf. Information Processing in Sensor Networks (IPSN), Apr. 2004.

[17] P. Ishwar, A. Kumar, and K. Ramchandran, "Distributed sampling for dense sensor networks: A bit-conservation principle," in Proc. Int. Conf. Information Processing in Sensor Networks (IPSN), Palo Alto, CA, Apr. 2003.

[18] P. Marziliano and M. Vetterli, "Reconstruction of irregularly sampled discrete-time bandlimited signals with unknown sampling locations," IEEE Trans. Signal Process., vol. 48, no. 12, pp. 3462-3471, Dec. 2000.

[19] R. Cristescu and M. Vetterli, "On the optimal density for real-time data gathering of spatio-temporal processes in sensor networks," in Proc. Int. Conf. Information Processing in Sensor Networks (IPSN), Los Angeles, CA, Apr. 2005.

[20] Y. Sung, L. Tong, and H. V. Poor, "Sensor activation and scheduling for field detection in large sensor arrays," in Proc. Int. Conf. Information Processing in Sensor Networks (IPSN), Los Angeles, CA, Apr. 2005.

[21] D. Ganesan, S. Ratnasamy, H. Wang, and D. Estrin, "Coping with irregular spatio-temporal sampling in sensor networks," in Proc. ACM SIGCOMM, Jan. 2004, pp. 125-130.

[22] M. C. Vuran, Ö. B. Akan, and I. F. Akyildiz, "Spatio-temporal correlation: Theory and applications for wireless sensor networks," Comput. Netw., vol. 45, no. 3, pp. 245-259, Jun. 2004.

[23] Y. Rachlin, R. Negi, and P. Khosla, "Sensing capacity for discrete sensor network applications," in Proc. Int. Conf. Information Processing in Sensor Networks (IPSN), Los Angeles, CA, Apr. 2005.

[24] P. Zhao, C. Zhao, and P. G. Casazza, "Perturbation of regular sampling in shift-invariant spaces for frames," IEEE Trans. Inf. Theory, vol. 52, no. 10 , pp. $4643-4648$, Oct. 2006.

[25] D. S. Early and D. G. Long, "Image reconstruction and enhanced resolution imaging from irregular samples," IEEE Trans. Geosci. Remote Sens., vol. 39, no. 2, pp. 291-302, Feb. 2001.

[26] A. Nordio, C.-F. Chiasserini, and E. Viterbo, "The impact of quasiequally spaced sensor layouts on field reconstruction," in Proc. Int. Conf. Information Processing in Sensor Networks (IPSN), Cambridge, MA, Apr. 2007.

[27] S. M. Kay, Fundamentals of Statistical Signal Processing: Estimation Theory. Englewood Cliffs, NJ: Prentice-Hall, 1993.

[28] T. Strohmer, "Computationally attractive reconstruction of bandlimited images from irregular samples," IEEE Trans. Image Process., vol. 6, no. 4, pp. 540-545, Apr. 1997.

[29] P. Billingsley, Probability and Measure, 3rd ed. New York: Wiley, 1995.

[30] L. Li, A. M. Tulino, and S. Verdù, "Asymptotic eigenvalue moments for linear multiuser detection," Commun. Inf. Syst., vol. 1, no. 3, pp. 273-304, Sep. 2001.

[31] E. W. Weisstein, "Bell Number," MathWorld-A Wolfram Web Resource [Online]. Available: http://mathworld.wolfram.com/BellNumber.html 
[32] E. W. Weisstein, "Stirling number of the second kind," MathWorld—A Wolfram Web Resource [Online]. Available: http://mathworld.wolfram.com/StirlingNumberoftheSecondKind.html

[33] "Kronecker delta," [Online]. Available: http://en.wikipedia.org/wiki/ Kronecker_delta

[34] The On-Line Encyclopedia of Integer Sequences [Online]. Available: http://www.research.att.com/ njas/sequences/A001263

[35] I. Dumitriu and E. Rassart, "Path counting and random matrix theory," Electron. J. Combinatorics, vol. 10, no. 1, 2003.

[36] M. Brookes, The Matrix Reference Manual [Online]. Available: http:// www.ee.ic.ac.uk/hp/staff/dmb/matrix/intro.html 2005

[37] N. Dershowitz and S. Zaks, "Ordered trees and non-crossing partitions," Disc. Math, vol. 62, pp. 215-218, 1986.

[38] R. P. Stanley, Enumerative Combinatorics. Cambridge, U.K.: Cambridge Univ. Press, 1999, vol. 2.

[39] R. Bellman, Introduction to Matrix Analysis, 2nd ed. New York: McGraw-Hill, 1970.

[40] W. H. Press, S. A. Teukolsky, W. T. Vetterling, and B. P. Flannery, Numerical Recipes, 2nd ed. Cambridge, U.K.: Cambridge Univ. Press, 1997.

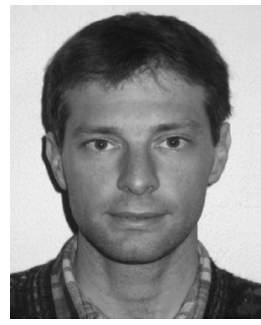

Alessandro Nordio (M'03) received the Laurea degree in telecommunications engineering from Politecnico di Torino, Turin, Italy, in 1998, and the Ph.D. degree from Ecole Politechnique Fédérale de Lausanne, in 2002.

He then joined the Department of Electronic Engineering of Politecnico di Torino where he is working as Postdoctoral Researcher. His research interests are in the field of signal processing, multiuser detection, space-time coding, sensor networks, and theory of random matrices.

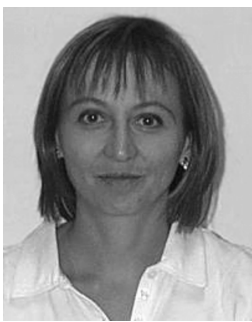

Carla-Fabiana Chiasserini (M'98) received the Laurea degree from the University of Florence, Florence, Italy, in 1996 and the Ph.D. degree from the Politecnico di Torino, Turin, Italy, in 2000.

From 1998 to 2003, she worked as a Visiting Researcher at the University of California at San Diego. She is currently an Associate Professor at Politecnico di Tornio.

Dr. Chiasserini is a member of the editorial board of the Ad Hoc Networks Journal (Elsevier) COMMUNICATIONS LETTERS since 2004. and has served as an Associate Editor of the IEEE

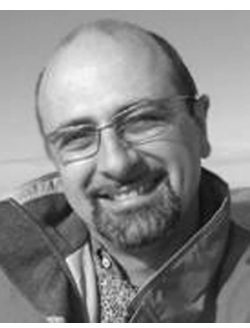

Emanuele Viterbo (M'95-SM'04) received the Laurea and $\mathrm{Ph} . \mathrm{D}$. degrees, both in electrical engineering, from the Politecnico di Torino, Turin, Italy, in 1989 and 1995 , respectively.

From 1990 to 1992, he was with the European Patent Office, The Hague, The Netherlands, as a Patent Examiner. He became Associate Professor at the Dipartimento di Elettronica, Politecnico di Torino, in 2005, and since November 2006, he has been a Full Professor at the Università della Calabria, Italy.

Dr. Viterbo was awarded a NATO Advanced Fellowship in 1997 from the Italian National Research Council. He is Associate Editor of the IEEE TRANSACTIONS ON INFORMATION THEORY, the European Transactions on Telecommunications, and the Journal of Communications and Networks. 